\title{
Phytohormones Regulate the Non-Redundant Response of $\omega-3$ Fatty Acid Desaturases to Low Temperatures in Chorispora Bungeana
}

Yulan Shi ( $\square$ sylbaby15@lzb.ac.cn )

Northwest Institute of Eco-Environment and Resources https://orcid.org/0000-0002-5657-819X

\section{Sizhong Yang}

Northwest Institute of Eco-Environment and Resources

\section{Xiule Yue}

Lanzhou University School Of Life Sciences

\section{Zhixing Zhao}

Lanzhou University School Of Life Sciences

\section{Lizhe An}

Beijing Forestry University College of Forestry

\section{Research Article}

Keywords: Chorispora bungeana (C. bungeana), w-3 FAD genes, trienoic fatty acids (TAs), phytohormones, low temperatures

Posted Date: July 27th, 2021

DOI: https://doi.org/10.21203/rs.3.rs-719872/v1

License: (c) (i) This work is licensed under a Creative Commons Attribution 4.0 International License. Read Full License 


\section{Abstract}

To explore the contribution of $\omega-3$ fatty acid desaturases (FADs) to cold stress response in a special cryophyte, Chorispora bungeana (C. bungeana), two plastidial $\omega-3$ FAD genes (CbFAD7 and CbFAD8) were cloned and verified in a Arabidopsis fad7fad 8 mutant, before being compared with the microsomal $\omega-3$ FAD gene ( $C b F A D 3)$ on expression profile. Though these genes were expressed in all tested tissues of $C$. bungeana, CbFAD7 and CbFAD 8 have the highest expression in leaves, while CbFAD3 was mostly expressed in non-green tissues. Low temperatures $\left(4,0\right.$ and $\left.-4{ }^{\circ} \mathrm{C}\right)$ resulted in significant increases in trienoic fatty acids (TAs, mainly $\mathrm{C} 18: 3$ ), which were consistent with the non-redundant expression of $C b F A D 3$ and $C b F A D 8$ in suspension-cultured cells, and the coordination of $C b F A D 7$ and $C b F A D 8$ in leaves. Furthermore, the contribution of CbFAD8 increased as temperature decrease in the two tissues. Our data revealed that jasmonie acid and brassinosteroids participated in the cold-responsive expression of these genes in both tissues, and the pyhtohormone regulation in leaves was more complicated with the participation of abscisic acid and gibberellin. These results point to the hormone-regulated non-redundant contribution of $\omega-3$ CbFADs to maintain appropriate level of TAs under low temperatures, which help $C$. bungeana survive in cold environments.

\section{Key Message}

This is the first evidence that jasmonie acid and brassinosteroids participate in the non-redundant response of $\omega-3$ fatty acid desaturases to low temperatures in Chorispora bungeana

\section{Introduction}

Low temperature is one of the major environmental stresses influencing plant performance and distribution. To withstand this stress, plants have developed various protective mechanisms that prevent and reduce the negative impacts (Iba 2002; Khan et al. 2017). Cell membranes, serving as a boundary and an active interface between cells/organelles and their environment, are the major targets of low temperature acclimation (Iba 2002; Shi et al. 2008; Khan et al. 2017). Membrane integrity and function, affected by temperature variation, can be maintained through the modification of fatty acids (Mikami and Murata 2003; Shi et al. 2008). In fact, the content of TAs, represented mainly by C18:3, changes to a certain extent in response to low temperatures, thus help maintaining membrane fluidity and structure (Matsuda et al. 2005; Upchurch 2008; Karabudak et al. 2014).

The production of TAs is catalyzed by $\omega-3$ FADs through adding an unsaturated double bond to the $\omega-3$ position of dienoic fatty acids (Iba 2002). It is known that $\omega$-3 FADs are one kind of acyl-lipid desaturases, which could be classified into two types according to cellular localization: The plastid-type desaturase (FAD7 and FAD8) is localized in plastid membranes (Iba et al. 1993; Gibson et al. 1994) and the microsome-type desaturase (FAD3) is localized in the endoplasmic reticulum (Arondel et al. 1992). As one of the most important factors for plant cold response (Iba and Kodama 1994), the expressions of $\omega-3$ FAD genes have been researched in various plants. The first finding was come from maize leaves, which 
showed an increase in ZmFAD8 mRNA accompanied by a decrease in ZmFAD7 mRNA under $5^{\circ} \mathrm{C}$ exposure (Berberich et al. 1998). Then, the cold-responsive expression of each plastidial and microsomal w-3 FAD gene was studied in some other plants, such as birch (Martz et al. 2006), Descurainia sophia (Tang et al. 2007), purslanen (Teixeira et al. 2010), soybean (Román et al. 2012), Arabidopsis (Shi et al. 2011; Román et al. 2015), safflower (Guan et al. 2014), Gossypium raimondii (Liu et al. 2015), Medicago truncatula (Zhang et al. 2018) and rice (E et al. 2019). All these researches were performed in plant leaves exposure to chilling temperatures $\left(2\right.$ to $\left.16^{\circ} \mathrm{C}\right)$, and found an increased FAD 8 mRNA accompanied by a decreased FAD7 or FAD3 mRNA. However, little attention has been paid to the typical cold-tolerant plants (i.e. cryophytes) as well as severe low temperatures (e.g. subzero temperatures). Therefore, it is unclear the difference between cryophytes and the other plant species in the contribution of $\omega-3$ FADs to cold stress response.

Besides that, though some phytohormones, for example abscisic acid (ABA), salicylic acid (SA), jasmonie acid (JA), brassinosteroids (BRs) and gibberellin (GA) were thought to be the signal molecules involved in plant cold response (Shi et al. 2015; Verma et al. 2016; Eremina et al. 2016; Khan et al. 2017; Ku et al. 2018), whether these hormones participate in the cold-responsive expressions of $\omega-3$ FAD genes doesn't attract enough attention. There was only one direct evidence confirmed that JA partially participates in the chilling-induced regulation of $\omega-3$ FAD genes in Arabidopsis (Shi et al. 2011). Recently, a study from Arabidopsis leaves indicated that AtFAD7 protein levels decreased upon ABA treatment, while AtFAD8 protein levels increased upon cold or JA exposure (Soria-García et al. 2019). However, the new findings haven't directly proved the regulation of ABA and JA on the cold response of AtFAD7 and AtFAD8. So far, the phytohormones that transmitted the low temperature signal to $\omega-3$ FAD genes and induced their expression are still ambiguous. As a result, research is far from having an integrated view of how $\omega-3$ FADs in typical cold-tolerant plants respond to low temperatures and through which mechanism this role is actually executed.

C. bungeana is a perennial cruciferous cryophyte, having close genetic relationship with Arabidopsis (Zhao et al. 2012). It inhabits periglacial regions (about 3800-3900 m), experiencing low temperature and freeze-thaw cycle all year round. Our previous studies found that $C$. bungeana possesses certain physiological and molecular mechanisms (Fu et al. 2006; Zhang et al. 2006; Shi et al. 2008; Di et al. 2009; Wu et al. 2009; Zhang et al. 2009; Zhao et al. 2012; Yue et al. 2019) instead of special morphological characteristics (Ayiturhan et al. 1998), which help it surviving in the extreme cold. Moreover, we found that the cold tolerance of $C$. bungeana suspension-cultured cells was closely related to the marked increased C18:3 (Shi et al. 2008), i.e. the product of $\omega-3$ CbFADs. However, the contribution of each $\omega-3$ CbFADs to the cold-induced C18:3 accumulations as well as the cold tolerance are unknown in $C$. bungeana.

In this work, we cloned two plastidial $\omega-3$ FAD genes from $C$. bungeana and studied the cold-responsive expression of different $\omega-3$ CbFAD genes to understand how they respond to low temperatures. Meanwhile, the relationship between the cold-responsive gene expression and the phytohormones, including $A B A, S A, J A, B R s$ and $G A 3$, were confirmed through the exogenous addition, the inhibitory 
effects and the concentration detection of corresponding hormones. The experiments were performed on C. bungeana cell suspension cultures as well as on the leaves of regenerated plants to extend the analysis to a tissue system. The data suggest the existence of phytohormone regulation on $\omega-3 \mathrm{CbFADs}$ in $C$. bungeana affecting specific isoforms in both plastid and microsome to maintain appropriate level of TAs under low temperatures and contribute to cold tolerance.

\section{Materials And Methods}

\section{Plant material and experiment treatments}

The suspension-cultured cells and the regenerated plants of $C$. bungeana were prepared as described by Shi et al. (2008) and Fu et al. (2006), respectively. Regenerated plants having 3-5 cm long roots were used for experiments. Arabidopsis seeds of Col-O (WT), fad7fad8 mutant (N8036, NASC, UK), and complemented fad7fad8 mutants (F7 and F8) were cultivated as our previous procedure (Shi et al. 2011).

For cold treatments, $C$. bungeana cell suspensions and regenerated plants were exposed to $4{ }^{\circ} \mathrm{C}, 0{ }^{\circ} \mathrm{C}$ or $-4{ }^{\circ} \mathrm{C}$ for $24 \mathrm{~h}$, respectively. For low-temperature germination, Arabidopsis seeds planted on MS medium were exposed to $15^{\circ} \mathrm{C}$ for 4 weeks. For exogenous hormone treatments, the cell suspensions and regenerated plants were moved to $1 / 2 \mathrm{MS}$ medium with $100 \mu \mathrm{M} \mathrm{JA}, 0.5 \mu \mathrm{M} \mathrm{BRs}, 100 \mu \mathrm{M} \mathrm{SA}, 100 \mu \mathrm{M}$ $\mathrm{ABA}$, or $100 \mu \mathrm{M} \mathrm{GA}_{3}$ for $24 \mathrm{~h}$, respectively. For phytohormone inhibitions, the cell suspensions and regenerated plants were moved to $1 / 2 \mathrm{MS}$ medium with $10 \mu \mathrm{M}$ sodium diethyldithiocarbamate trihydrate (DIECA), $10 \mu \mathrm{M}$ propiconazole (Pcz), $10 \mu \mathrm{M}$ fluricbne (Flu), or 10 $\mu \mathrm{M}$ paclobutrazol (Pac) for $24 \mathrm{~h}$, respectively. To ensure the hormone/inhibitor application on leaves, the over ground part of each plant was sprayed with corresponding solution after moving, and the excess liquid was blotted by cotton balls. The $C$. bungeana cells and leaves were collected at different time spots according to the experimental design in each treatment, while the leaves of different Arabidopsis lines were collected from 5 weeks old plants under normal growth conditions. All the collected samples were stored at $-80^{\circ} \mathrm{C}$ until use.

\section{RNA isolation and cDNA synthesis}

Total RNAs of $C$. bungeana were isolated from the suspension-cultured cells or the different tissues of regenerated plants $(0.1 \mathrm{~g}$ each) according to the standard procedure of Plant RNA Kit (Omega, USA). After being treated with RQ1RNase-free DNase (Promega, USA), the purity and integrity of total RNA were assessed by UV spectrophotometry and agarose gel electrophoresis. Then, the qualified total RNAs were employed in reverse transcription reaction by using PrimeScript ${ }^{\mathrm{TM}}$ Reverse Transcriptase (Takara, Japan) and Oligo(dT) ${ }_{15}$ primer (Takara, Japan) following the manufacturer's instruction. Reverse-transcribed cDNA samples were stored at $-20^{\circ} \mathrm{C}$ until further use.

\section{Cloning and bioinformatics analysis of CbFAD7 and CbFAD8}


A 732-bp fragment of CbFAD7 and a 982-bp fragment of CbFAD8 were cloned from $C$. bungeana by using degenerate primers ( $\mathrm{P} 1$ and $\mathrm{P} 2$ for CbFAD7; $\mathrm{P} 3$ and $\mathrm{P} 4$ for CbFAD8; Table $\mathrm{S} 1$ ) designed basing on the conserved domain database from tobacco, Brassica napus and Arabidopsis. Amplification of $5^{\prime}$ and 3' ends of $C b F A D 7$ and $C b F A D 8$ were accomplished using specific primers (P5-P8 for CbFAD7; P9-P12 for CbFAD8; Table S1) and SMARTer ${ }^{\text {TM }}$ RACE cDNA Amplification Kit (Clontech, Japan). Full-length cDNA of CbFAD7 and CbFAD 8 were gotten by using specific primers (P13 and $\mathrm{P} 14$ for CbFAD7; $\mathrm{P} 15$ and $\mathrm{P} 16$ for CbFAD8; Table S1). PCR products were cloned into the PMD-18T vector (Takara, Japan) and sequenced by GENEWIZ Inc. (Suzhou, China). Then these sequences were analyzed by DNAman 5.2.9, MEGA 6.06 and ClustalX 1.83 software. The prediction of transmembrane domain and transit peptide were analyzed by the online server program TMHMM (http://www.cbs.dtu.dk/services/TMHMM/) and TargetP (http://www.cbs.dtu.dk/services/TargetP/), respectively.

\section{Complementation of CbFAD7 and CbFAD8 in Arabidopsis mutant}

The coding region of CbFAD7 amplified using specific primers (P17and P18; Table S1), were cloned within the Xbal-Sacl site of the binary vector pBI121 to replace the GUS gene and construct the recombinant plasmid, pBI121-CbFAD7, under the control of CaMV 35S promoter. In the same way, the recombinant plasmid $\mathrm{pBI} 121-C b F A D 8$ was constructed with the coding region of $C b F A D 8$ amplified using specific primers (P19 and P20; Table S1). Then, the two recombinant plasmids and the Arabidopsis seeds of fad7fad8 mutant were sent to Shanghai Weidi Biotechnology Co., Ltd (Shanghai, China) to get the complemented mutants, F7 and F8. The transgenic Arabidopsis plants were generated through the floral dip by Agrobacterium-mediated transformation. Positive transgenic lines of F7 and F8 exhibiting 3:1 segregation ratio were identified by PCR using specific primers (P17 and P18 for CbFAD7; P19 and P20 for $C b F A D 8$; Table $\mathrm{S} 1$ and Fig. S1), respectively. The homozygous lines were obtained by backcrossing or self-pollination, and the expression level of $C b F A D 7$ and $C b F A D 8$ were verified by qRT-PCR using specific primers (P23 and P24 for CbFAD7; P25 and P26 for CbFAD8; Table S1 and Fig. S1), respectively. IPT2 (AT2G27760) was taken as the house keeping gene (Cheng et al. 2017) using primers P29 and P30 (Table S1). Three independent homozygous T3 transgenic lines of F7 and F8 showing higher expression levels were used in the experiments, respectively.

\section{Quantitative real-time PCR}

The SYBR Green I (Takara, Japan) assay and the Real-Time PCR System (Mx3000P, Agilent Stratagene, USA) were used for detecting the expression of CbFAD3, CbFAD7 and CbFAD 8 in $C$. bungeana. The housekeeping gene, CbACT (AY825362), was used as a control for the stable expression (Di et al. 2009; Wu et al. 2009; Zhang et al. 2009; Shi et al. 2018). The amplification specificity of the primers (P21 and P22 for CbFAD3; P23 and P24 for CbFAD7; P25 and P26 for CbFAD8; P27 and P28 for CbACT; Table S1) were checked by gel electrophoresis before real-time PCR. The amplification condition was as follow: 95 ${ }^{\circ} \mathrm{C}$ for $30 \mathrm{sec}$, and 40 cycles of $95{ }^{\circ} \mathrm{C}$ for $5 \mathrm{~s}$ and $58{ }^{\circ} \mathrm{C}$ for $34 \mathrm{~s}$. This was followed by $15 \mathrm{~s}$ at $95{ }^{\circ} \mathrm{C}, 60 \mathrm{~s}$ at $60^{\circ} \mathrm{C}$ and $15 \mathrm{~s}$ at $95^{\circ} \mathrm{C}$ (determination of melting curve). PCR data were obtained from three 
independent biological samples for each experiment. The relative gene expression (F) was normalized against the housekeeping gene according to the formula:

$$
\mathrm{F}=\frac{\left(\mathrm{E}_{\text {target }}\right)^{\Delta \mathrm{Ct}_{\text {target }} \text { (control-sample) }}}{\left(\mathrm{E}_{\text {housekeeping }}\right)^{\Delta \mathrm{Ct}_{\text {housekeeping }} \text { (control-sample) }}}
$$

reproducibility mathematical model (Pfaff 2001).

, which was regarded as a high accuracy and

\section{Extraction and analysis of total fatty acids}

The cell suspensions and the plant leaves of $C$. bungeana as well as the leaves of Arabidopsis $(2 \mathrm{~g}$ each) were grinded with liquid N2, respectively. Lipids and total fatty acids of each sample were prepared and analyzed as our previous reports (Shi et al. 2008; Shi et al. 2011). Fatty acid methyl esters of each sample were analyzed by GC-MS (6890N-5975C, Agilent, USA) fitted with a capillary column (Agilent DB-FFAP, 30 $\mathrm{m} \times 0.25 \mathrm{~mm} \times 0.5 \mu \mathrm{m}$ ) according to our previous procedure (Shi et al. 2018). Fatty acid data were obtained from five independent biological samples for each experiment.

\section{Extraction and analysis of phytohormones}

The $C$. bungeana suspension-cultured cells and leaves $(0.5 \mathrm{~g}$ each) were sent to Genepioneer Biotechnologies (Nanjing, China) to detect the concentration of JA, BRs, ABA and GA3, respectively. Phytohormones were extracted through grinding and organic solvent extraction, and then analyzed using Plant JA ELISA Kit (Sinobestbio, China), Plant BRs ELISA Kit (Sinobestbio, China), Plant ABA ELISA Kit (Sinobestbio, China) and Plant GA3 ELISA Kit (Sinobestbio, China), respectively. During the doubleantibody sandwich ELISA, the absorbance (OD value) was detected by microplate reader (Infinite F50, Tecan, SWIT), and the concentration was calculated through standard curve method. Phytohormone data were from five biological replicates for each experiment.

\section{Statistical analysis}

Statistical analysis was performed using the method of one-way ANOVA, followed by Duncan's multiple range test at the $\mathrm{P}<0.05$ or $\mathrm{P}<0.01$ levels.

\section{Results}

\section{cDNA isolation and sequence analysis of CbFAD7 and CbFAD8from $C$. bungeana}

Based on the nucleotide sequences of known plastidial $\omega-3$ FADgenes, two pairs of degenerate primers were designed and used for getting conserved sequences. After RACE PCR reactions and full length verification, two full length cDNA of 1805 and 1563 bp were obtained and designated as CbFAD7 (KY069282) and CbFAD8 (KY069283), respectively. CbFAD7 contains an ORF encoding a predicted protein (CbFAD7) of 439 aa, which corresponds to a calculated molecular mass of $50.2 \mathrm{kDa}$ and a pl of 
7.89. The deduced CbFAD7 sequence displayed significant similarity to other known plant FAD7 sequences, exhibiting the highest identity (85\%) to Brassica napus BnFAD7 (FJ985690). CbFAD8 contains an ORF encoding a predicted protein (CbFAD8) of 397 aa, which corresponds to a calculated molecular mass of $45.6 \mathrm{kDa}$ and a pl of 8.92. The deduced CbFAD8 sequenceshowed remarkable similarity to other known plant FAD8 sequences and the highest identity (84\%) to the Arabidopsis AtFAD8(NM120640). These data suggest that CbFAD7 and CbFAD8 cloned from $C$. bungeana encode the plastidial $\omega-3$ FADs.

Analysis of the deduced CbFAD7 and CbFAD8 sequences with targeting prediction tools, showed the 50 and 60 aa N-terminal transit peptides with the characteristic features of chloroplast targeting peptide (Arrowed in Fig. 1a), respectively, predicting a subcellular localization in chloroplasts. Alignment of the two deduced protein sequences found there were three conserved histidine clusters (HDGCH, HXXXXXHRTHH and HHXXXXHVIHH, asterisked in Fig. 1a) and four transmembrane domains (TMD, underlined in Fig. 1a), suggesting that the predicted proteins of $C b F A D 7$ and CbFAD 8 are chloroplast membrane-bound $\omega-3$ FADs.

To elucidate the phylogenetic relationship, the predicted proteins of $C b F A D 7, C b F A D 8$ and $C b F A D 3$ were included in a dendrogram representing the known plant $\omega-3$ FADs for comparison. CbFAD7 (ARL62096) and CbFAD 8 (ARL62097) were positioned in the group corresponding to plastidial $\omega$-3 FADs, whereas CbFAD3 (AKN35208) was grouped with those of microsomal location (Fig. 1b).

\section{The functionality of $C b F A D 7$ and $C b F A D 8$ were verified in Arabidopsis mutant}

To determine the functionality of $C b F A D 7$ and $C b F A D 8$, the ORF of the two genes were expressed in double fad7fad8 mutants under the CaMV 35 S promoter of pBI121 vector, respectively. The fatty acids of leaf lipids showed that though the $\mathrm{C} 18: 3$ contents in the complemented mutants F7 and F8 were still lower than that in WT plants, they were markedly higher than that in fad7fad8 mutants; moreover, the C18:3 content in F7 lines was much higher than that in F8 lines, which may indicate a stronger conversion effect of CbFAD7 on C18:3 (Fig. 2a). Being exposed to $15^{\circ} \mathrm{C}$, the germination rates of $\mathrm{F} 7$ and F8 seeds were significantly higher than that of fad7fad 8 mutant seeds, and close to that of WT seeds (Fig. 2b). These data confirmed that both CbFAD7 and CbFAD 8 were functional plastical $\omega-3$ FAD genes.

\section{The tissue-specific and cold-responsive expressions of $\omega-3$ CbFAD genes in $C$. bungeana}

The expression profiles of plastical and microsomal $\omega-3$ CbFAD genes were analyzed in the cell suspensions and the regenerated plants of $C$. bungeana. Both $C b F A D 7$ and $C b F A D 8$ were highly expressed in leaves and lowly expressed in roots (Fig. 3a), showing the tissue-specific expression parttern with the characteristic feature of plastidial $\omega-3$ FAD genes. However, the expression level of CbFAD7 in suspension-cultured cells was just lower than that in leaves, while the expression level of CDFAD 8 in cultured cells was as low as that in roots. CbFAD3 were highly expressed in non-green tissues, e.g. suspension-cultured cells and roots, and lowly expressed in stems and leaves (Fig 3a), showing the tissue-specific expression pattern with the characteristic feature of microsomal $\omega-3$ FAD genes. 
Given that microsomal and plastical $\omega-3$ CbFAD genes mostly expressed in suspension-cultured cells and plant leaves, respectively, the cold-responsive expression of these genes were detected in the two tissues. In cell suspensions, the expression of $C b F A D 3$ rapidly increased at 4 (6.3-fold) and $0{ }^{\circ} \mathrm{C}(5.7-$ fold), and decreased at $-4{ }^{\circ} \mathrm{C}$ (Fig. $3 \mathrm{~b}$ ). Conversely, the expression of CbFAD 8 was almost no change at $4{ }^{\circ} \mathrm{C}$, but sharply increased at 0 (13.1-fold) and $-4{ }^{\circ} \mathrm{C}$ (27.7-fold) (Fig. 3b). In fact, the increases in $C b F A D 3$ and $C b F A D 8$ mRNA all peaked at being treated for $3 \mathrm{~h}$, and were accompanied by a decrease in $C b F A D 7$ mRNA at different low temperatures (Fig. 3b). Being compared with cell suspensions, the expression pattern of $C b F A D 3$ and $C b F A D 7$ in plant leaves interchanged, the former decreased at all tested low temperatures, the latter rapidly increased at 4 (4.1-fold) and $0{ }^{\circ} \mathrm{C}(2.6-f o l d)(F i g .3 b)$. Like that observed in cultured cells, the up-regulation of leaf $C b F A D 8$ was only induced by severe low temperatures, with the increase of 3.4-fold at $0{ }^{\circ} \mathrm{C}$ and 6.7 -fold at $-4{ }^{\circ} \mathrm{C}$ (Fig. 3b). In this case, the cold-induced increase in CbFAD7 and CbFAD 8 mRNA peaked at being treated for 3 and $6 \mathrm{~h}$, respectively. On the whole, the expression of $\omega-3$ CbFAD genes presented a non-redundant pattern in respond to different low temperatures.

\section{The hormone and inhibitor-responsive expressions of $\omega-3$ CbFAD genes in $C$. bungeana}

The expression of $\omega-3$ CbFAD genes respond to various exogenous hormones were studied in the cell suspensions and the plant leaves of $C$. bungeana. In suspension-cultured cells, the expression of $C b F A D 3$ was up-regulated by ABA, SA and BRs, but down-regulated by JA and GA3; among these regulations, the BRs-induced up-regulation (peaked at being treated for 3 h, 6.2-fold) was similar to those induced by chilling temperatures, and the JA-resulted down-regulation was in accord with that reduced by subzero temperatures (Fig. 3a and 4a). Although the expression of CbFAD7 was increased by ABA, SA and GA3, the decreased expression regulated by BRs and JA were consistent with the cold-reduced ones (Fig. 3a and 4a). The increase in CbFAD 8 mRNA was induced by all tested hormones except GA3, however, only the JA- and the BRs-induced increases (9.3-fold and 8.1-fold, respectively) peaked at being treated for $3 \mathrm{~h}$ and resembled those induced by severe low temperatures (Fig. 3a and 4a). Unlike that be found in cell suspensions, the expression of $C b F A D 3$ in leaves was down-regulated by all tested hormones with different dynamics, each of which approached the cold-reduced ones in varied degrees (Fig. 3b and 4b). Meanwhile, the expression of leaf $C b F A D 7$ was increased by BRs, JA and GA3, but reduced by ABA; among these changes, the increases induced by BRs and GA3 (1.6-fold and 1.9-fold, respectively) peaked at being treated for $3 \mathrm{~h}$ and were like those induced by chilling temperatures (Fig. 3b and 4b). Similarly, the expression of leaf CbFAD8 was also induced by three hormones (ABA, BRs and JA) and reduced by one (GA3), however, only the increases induced by ABA and JA (4.3-fold and 3.6-fold, respectively) peaked at being treated for $6 \mathrm{~h}$ and resembled those induced by severe low temperatures (Fig. $3 \mathrm{~b}$ and $4 \mathrm{~b}$ ). The Pearson correlations between the cold- and the hormone-responsive expressions of $\omega-3$ CbFADgenes (Table 1 and 2) indecated that JA and BRs may participate in the cold-responsive expresssions of thesegenes in both cultured cells and plant leaves, while ABA and GA3 may only participate in the coldresponsive gene expressions in leaves. 
To further confirm the participations of these hormones, the corresponding inhibitors were used. Data showed that in cell suspensions (Fig. 5a), the cold-induced expression of CbFAD3 was completely inhibited by Pcz (a synthetic inhibitor of BRs), but the inhibition could be partly eliminated by DIECA (a synthetic inhibitor of $\mathrm{JA}, 45.1 \%$ ); the cold-reduced expression of $C b F A D 7$ was mainly eliminated by DIECA (65.8\%) and partially eliminated by Pcz (32.9\%), but completely eliminated by the synergism of them; in contrast, the cold-induced expression of CbFAD8 gene was mostly inhibited by either DIECA (79.9\%) or Pcz (78.9\%), but completely inhibited by the cooperation of them. In plant leaves (Fig. 5b), the cold-reduced expression of CbFAD3 was completely eliminated by the coordination of Pcz, DIECA, Pac (a synthetic inhibitor of GA3) and Flu (a synthetic inhibitor of $A B A$ ), and the synergistic effect of Pcz and DIECA play a major role (79.0\%); the cold-induced expression of $C b F A D 7$ can be mainly inhibited by Pcz $(66.0 \%)$ and partially inhibited by Pac (33.9\%), but completely inhibited by the collaboration of them; likewise, the cold-induced expression of CbFAD 8 can be mostly inhibited by DIECA (73.1\%) and partially inhibited by Flu $(29.9 \%)$, but completely inhibited by the combined effect of them.

All these results suggested that in $C$. bungeana cultured cells, the cold-responsive expression of $C b F A D 3$ may be induced by BRs and reduced by JA; the synergism of JA and BRs may decrease CbFAD7 mRNA and increase $C b F A D 8$ mRNA in response to low temperatures, however, the over-high concentration of BRs can in turn inhibit the expression of CbFAD8 (Fig. 3b, 6b and S2). In C. bungeana leaves, the coldreduced expression of $C b F A D 3$ was regulated by the cooperation of BRs, JA, GA3 and ABA; while the coldinduced expressions of $C b F A D 7$ and $C b F A D 8$ were regulated by the coordination of BRs and GA3 as well as $J A$ and $A B A$, respectively.

\section{The level of related phytohormones in $C$. bungeanaduring low-temperature treatments}

To provide more evidence to the hormone-regulated cold response, the content changes of related phytohormones were detected in the cell suspensions and the plant leaves of $C$. bungeana at different low temperatures. Being treated at $0{ }^{\circ} \mathrm{C}$, the levels of all tested pyhtohormones presented a rapid and two-peaks increase in both tissues (Fig. 6a and 7a). In cultured cells, the level of BRs peaked at 1 (1.7fold) and $3 \mathrm{~h}$ (1.5-fold), while that of JA peaked at 1.5 (2.6-fold) and $3 \mathrm{~h}(2.4$-fold). In plant leaves, the content changes of BRs and JA were the same as or similar to those in cultured cells, except the peak times of JA increase ( 2 and $4 \mathrm{~h}$ ) were a little later; at the same time, the level of GA3 peaked at 0.5 (1.8fold) and $2.5 \mathrm{~h}$ (2.3-fold), and that of ABA peaked at 1 (1.6-fold) and $3 \mathrm{~h}$ (1.9-fold). Overall, the coldinduced hormone increases, especially the first peaks, were before the cold-responsive expressions of corresponding $\omega-3$ CbFAD genes (Fig. 3b, 6a and 7a); furthermore, the content increases in synergistic pairs, such as JA and ABA, or BRs and GA3, presented a peak stagger trend to avoid redundant effect at the same temperature (Fig. 6a and 7a).

Actually, all tested low temperatures could induce the content increases in these phytohormones, but the increment of the same phytohormone was varied (Fig. $6 \mathrm{~b}$ and 7b). In suspension-cultured cells (Fig. 6b),

the level of BRs highly increased at $4{ }^{\circ} \mathrm{C}(2.6-$ fold $)$ and slightly increased at $0{ }^{\circ} \mathrm{C}(1.7$-fold); as for $\mathrm{JA}$, the highest content increment was found at $-4{ }^{\circ} \mathrm{C}\left(3.1\right.$-fold), and the increments at 4 and $0{ }^{\circ} \mathrm{C}$ were the same 
(2.6-fold). The cold-induced hormone increases in leaves were more regular than those in cultured cells (Fig. 7b): the content increment of BRs decreased as temperature decrease, conversely, that of JA increased; meanwhile, the level of GA3 showed the highest and the lowest increases at -4 (4.2-fold) and 0 ${ }^{\circ} \mathrm{C}$ (1.8-fold), respectively, while those of ABA presented in an opposite way (1.9-fold at $0{ }^{\circ} \mathrm{C}$ and 1.2 -fold at $-4{ }^{\circ} \mathrm{C}$ ). On the whole, the increment changes in these phytohormones, were consistent with the dynamic expression of corresponding $\omega-3$ CbFAD genes at different low temperatures; furthermore, the content increment of antagonistic pairs, for example GA3 and ABA, showed a reverse tendency in response to temperature variation, which was due to the trade-offs between plant growth and cold stress response (Fig. 3b, 6b and 7b).

\section{The level of TAs in $C$. bungeana during low-temperature and hormone-inhibitor treatments}

Considering that the cold-responsive expressions of $\omega-3$ CbFAD genes may affect the level of TAs, the C18:3 and the $\mathrm{C} 16: 3$ contents of total lipids were detected in the cell suspensions and the plant leaves of C. bungeana under different low temperatures. Because of lacking chloroplast, no C16:3 was detected in suspension-cultured cells (Fig. 8a). Data showed that the level of C18:3 was obviously induced by all tested low temperatures and reached the maximum at being treated for $12 \mathrm{~h}$ in both tissues; the $\mathrm{C} 18: 3$ content in cultured cells increased from about $20.6 \%$ to $46.2-55.0 \%$ of total fatty acids, and that in leaves increased from about $46.3 \%$ to $58.6-60.7 \%$ of total fatty acids (Fig. 8). Similarly, the C16:3 content of leaf lipids increased from about $2.8 \%$ to $4.3-5.2 \%$ of total fatty acids, and reached the maximum at being treated for $12\left(4\right.$ and $\left.0{ }^{\circ} \mathrm{C}\right)$ or $24 \mathrm{~h}\left(-4{ }^{\circ} \mathrm{C}\right)(\mathrm{Fig} .8 \mathrm{~b})$. In general, the marked increases in TAs were in accordance with but lagging behind the cold-responsive expressions of $\omega-3$ CbFAD genes (Fig. 3 and 8).

To further confirm that the phytohormones affect the cold-induced level of TAs through regulating $\omega-3$ CbFAD genes, the $\mathrm{C} 18: 3$ and the $\mathrm{C} 16: 3$ contents of total lipids were also detected in the cell suspensions and the plant leaves of $C$. bungeana under hormone-inhibitor treatments. In suspension-cultured cells, the cold-induced increase in C18:3 content was completely inhibited by the synergism of DIECA and Pcz, but partially inhibited by either of them (Fig. 8a); In plant leaves, the cold-induced increases in C18:3 and C16:3 contents were all completely inhibited by the synergistic effect of DIECA, Pcz, Pac and Flu, but partially inhibited by either of them (Fig. 8b). These results consisted with the inhibitor-responsive expressions of $\omega-3$ CbFAD genes (Fig. 5 and 8 ), suggesting that with the help of ABA and GA3 in leaves, JA and BRs participated in maintaining appropriate level of TAs in $C$. bungeana through regulating these genes under low temperatures.

\section{Discussion}

This work analyzed the behavior of the microsomal and plastidial $\omega-3$ FADs in $C$. bungeana at the level of both gene expression and fatty acid content, to determine how low temperature affected the contribution of each $\omega-3$ FAD to the synthesis of TAs, especially C18:3. Obviously, low temperatures resulted in significant transcriptional changes on each $\omega-3$ FAD, notably the sharp increased CbFAD 8 mRNA occurred in both suspension-cultured cells and the leaves from regenerated plants (Fig. 3b). FAD8 was originally 
identified as a cold-specific desaturase by phenotypic analysis of a fad3/fad7 double mutant from Arabidopsis that was capable of producing TAs only at low temperatures (McConn et al. 1994). From then on, the increased expression of FAD8 were found in many plant species at various chilling temperatures $\left(4-10{ }^{\circ} \mathrm{C}\right)$ (Berberich et al. 1998; Martz et al. 2006; Wang et al. 2006; Tang et al. 2007; Teixeira et al. 2010; Shi et al. 2011; Liu et al. 2015; Román et al. 2015), excepting a few different observations (Román et al. 2012; Guan et al. 2014; Zhang et al. 2018). In this work, the increased CbFAD8 mRNA were not induced by the chilling temperature $\left(4{ }^{\circ} \mathrm{C}\right)$, but by severe low temperatures $\left(0\right.$ and $\left.-4{ }^{\circ} \mathrm{C}\right)$, and the increments increased as temperature decreased in both tested tissues. The results from $C$. bungeana not only support the previous view that FAD 8 was a cold-specific desaturase, but also indicate that CbFAD8 is the major $\omega-3$ desaturase contributed to the synthesis of TAs in response to severe low temperatures, particularly subzero temperatures. Given that FAD8 plays an important role in the biosynthesis of plastid TAs (Zhang et al. 2005; Penfield 2008) required for the correct biogenesis and maintenance of chloroplasts (Routaboul et al. 2000) as well as the recovery from photo inhibition at low temperatures (Vijayan and Browse 2002; Tovuu et al. 2016), the induction of FAD8 under subfreezing temperatures may be essential for plant cold tolerance.

As for CbFAD3 and CbFAD7, the cold-responsive transcriptional changes presented a tissue-specific profile (Fig. 3b). During low temperature exposure, the CbFAD3 mRNA increased in suspension-cultured cells and decreased in plant leaves, while the expressions of CbFAD7 in the two tissues were just the opposite. Previous researches showed that even in the same type of tissue under a similar temperature, the expression profiles of $F A D 3$ and FAD7 were varied with plant species (Berberich et al. 1998; Martz et al. 2006; Tang et al. 2007; Shi et al. 2011; Zhang et al. 2011; Román et al. 2012; Karabudak et al. 2014; Ma et al. 2014; Yurchenko et al. 2014), which means some observations are consistent with ours but the others not. Although we don't know why these enzymes provide different contributions in distinct plant species, it is not surprising that the contributions of CbFAD3 and CbFAD7 in $C$. bungeana were proportional to their transcript abundance in distinct tissues (Fig.3).

In fact, the cold-induced increments of $C b F A D 3$ or $C b F A D 7$ mRNA decreased as temperature decreased, and formed a non-redundant complementation with the gradually increased CbFAD 8 mRNA in distinct tissues (Fig. 3b). In C. bungeana cell suspensions, the non-redundant coordination of $C b F A D 3$ and CDFAD 8 indicate the distinct TA needs under different low temperatures, for FAD3 affecting total TA levels and FAD8 affecting plastid TA levels (Zhang et al. 2005; Penfield 2008). The non-redundant cooperation of $F A D 7$ and $F A D 8$ was also found in Arabidopsis leaves at different temperatures $\left(8,22\right.$ and $30{ }^{\circ} \mathrm{C}$; Román et al. 2015). Though both FAD7 and FAD8 affect the synthesis of TAs in chloroplastic lipids, the lipid specificity of them are different (Soria-García et al. 2019): AtFAD7 prefer galactolipids, which are the major chloroplast lipids with higher TA content; while AtFAD8 prefer phosphatidylglycerol (Román et al. 2015), which has specific role in the stability of photosynthetic complexes (Wada and Murata 2007). Hence, the non-redundant cooperation of $C b F A D 7$ and $C b F A D 8$ in $C$. bungeana leaves may due to the distinct needs of photosynthetic activity and stability under different low temperatures. Nevertheless, the non-redundant expression of $\omega-3$ CbFAD genes maintained appropriate level of TAs, especially $C 18: 3$, in response to low temperatures (Fig. 8a). 
Through a series of verification, including exogenous hormone application (Fig. 4), correlation analysis (Table 1), inhibitor treatments (Fig. 5 and 8) and phytohormone detection (Fig. 6 and 7), we confirmed that the cold-responsive non-redundant expression of $\omega-3 \mathrm{CbFAD}$ genes were regulated by certain phytohormones, notably JA and BRs. JA is mainly responsible for the induction of FAD 8 under freezing/subfreezing temperatures, with the association of BRs in cell suspensions, and ABA in plant leaves; $\mathrm{BRs}$ is in charge of the induction of $C b F A D 3$ or $C b F A D 7$ under chilling/freezing temperatures, with or without the help of GA3 in distinct tissues. Moreover, both JA and BRs took part in the down-regulation of corresponding $\omega-3$ CbFAD gene in response to cold stress, with or without the participation of $A B A$ and GA3 in different tissues. These results not only provide new insights into our previous findings that JA partially mediates the chilling-induced transcription of $\omega-3$ FAD genes in Arabidopsis (Shi et al. 2012), but also agree with that JA may act as a core signal by interacting with other phytohormones to regulate the balance between plant growth and stress response. Among these interactive hormones, ABA may function synergistically with JA signaling to modulate cold-responsive genes (Hu et al. 2017; Kim et al. 2018; Wang et al. 2020); while the concentration of BRs decides it in synergism or antagonism with JA during stress defense (Yang et al. 2019); GA usually inhibits JA signaling, but in some cases, the synergistic effect of them is also exist (Hu et al.2017; Yang et al. 2019; Wang et al. 2020). The interactions of these phytohormones with JA all consist with the observations from $C$. bungeana. A recent study showed that in Arabidopsis leaves, AtFAD8 protein levels increased upon cold or JA exposure, but did not respond to ABA (Soria-García et al. 2019). All the findings indicate that though the interactive hormones of JA signaling may vary with tissues and plant species, JA did regulate the cold induction of FAD8 in both $C$. bungeana (cryophyte) and Arabidopsis (modal plant), which may be common in all plant species.

BRs are another important pyhtohormone took part in the cold-responsive expressions of $\omega-3$ FAD genes in $C$. bungeana. It is confirmed that BRs can improve frost tolerance by promoting GA biosynthesis and interplaying with GA at the signaling level (Ramirez and Poppenberger 2020). The findings provide support to our observation that the cooperation of BRs and GA3 participated in the cold-responsive expressions of $C b F A D 3$ and $C b F A D 7$ in $C$. bungeana leaves. Besides, BRs may participate in drought or cold stress acclimation by three interconnected mechanisms, one of which is in communication with $A B A$ signaling (Bulgakov and Avramenko 2020). This may explain the synergistic effect of BRs and ABA on the cold-responsive down-regulation of leaf $C b F A D 3$. However, because there was no report about the function of BRs on the cold-responsive expressions of $\omega-3$ FAD genes, we cannot predict that BRs also regulates the cold induction of $F A D 3$ or $F A D 7$ in other plant species before further research.

It is worth to note that the interactions between different pytohormones on $\omega-3 \mathrm{CbFAD}$ genes suggest each of the genes having the ability in response to multiple hormones (Fig. 4-8; Table 1). A similar phenomenon from Arabidopsis showed that the expression of AtFAD3 was regulated through the synergistic and antagonistic interactions of auxin, cytokinin and ABA during plant development (Matsuda et al. 2001). These results reflect the existence of various promoter cis-elements combined with different transcription factors (TFs) from corresponding hormone signaling. A G-box-like motif was first found in the promoter of AtFAD7 in Arabidopsis roots, which was required by JA-responsive expression (Nishiuchi 
et al. 1999). After that, no hormone-responsive element was confirmed in the promoter of $\omega-3$ FAD genes. Until recently, the multiple ABA responsive elements (ABREs) were found in the promoter of microalgae $\omega$-3 FAD gene (Norlina et al. 2020). However, the data is still too limited to have an integrated understanding.

As another necessary factor for the hormone regulation on $\omega-3$ FAD genes, TFs have been studied at recent years. On the one hand, some TFs for regulating the expression of $\omega-3$ FAD genes were verified: the expression of FAD3 is up-regulated by bZIP67 in Arabidopsis seeds (Mendes et al. 2013), but downregulated by MaMYB4 in banana fruits (Song et al. 2019) and by HD in soybean (Jo et al. 2021); WIPK is involved in wound-responsive expression of AtFAD7 gene in transgenic tobaccos (Kadoma et al. 2000). On the other hand, it is confirmed in Arabidopsis that some TFs (such as WRKYs, MYCs, bHLHs and ICEs) occurred at the downstream of cold-induced JA signaling (Hu et al. 2017; Wang et al. 2020), while some others (for example bZIPs) belong to the cold-responsive BR and/or ABA signaling pathways (Banerrjee and Roychoudhury 2017; Ramirez and Poppenberger 2020). Athough these relevant information pieces are incomplete, if we piece them together, it is not difficult to speculate that through the common or distinct TFs combined with corresponding promoter elements, JA and BRs as well as ABA and GA3 achieve the synergistic or antagonistic regulation on $\omega-3$ CbFAD genes, which result in non-redundant enzyme cooperation to maintain appropriate level of TAs and then help $C$. bungeana survive in cold environments.

\section{Declarations}

\section{Acknowledgments}

The authors would like to thank Dr. Tingting Fan (Hefei University of Technology) for technological help and Dr. Binquan Chu (Zhejiang University) for fatty acid analysis. This research has been funded by National Natural Science Foundation of China (31200278 and 32071482).

\section{Author contribution statement}

YS and LA conceived and designed research; $Y S$ and $X Y$ conducted experiments; SY and ZZ contributed to material preparation; $Y S$ analyzed data and wrote the manuscript; $X Y$ and LA provided suggestions and revised the manuscript. All authors read and approved the manuscript.

\section{Data availability}

The DNA and protein sequences generated during and/or analysed during the current study are available in the NCBI Genbank repository (https://www.ncbi.nlm.nih.gov/nuccore/or https://www.ncbi.nlm.nih.gov/protein/). The other datasets generated during and/or analysed during the current study are available from the corresponding author on reasonable request.

\section{Compliance with ethical standards}


Conflict of interest The authors declare that they have no competing interests.

\section{References}

1. Arondel V, Lemieux B, Hwang I, et al (1991) Map-based cloning of a gene controlling omega-3 fatty acid desaturation in Arabidopsis. Science 258: 1353-1355. https://doi.org/10.1126/science. 1455229

2. Ayiturhan, Tan D, Li Z et al (1998) The relationship between the structures of vegetative organs in Chorispora bungeana and its environment. J Xinjiang Agric Univ 21: 273-277.

3. BanerjeeA, Roychoudhury A (2017) Abscisic-acid-dependent basic leucine zipper (bZIP) transcription factors in plant abiotic stress. Protoplasma 254: 3-16. https://doi.org/10.1007/s00709-015-0920-4

4. Berberich T, Harada M, Sugawara K et al (1998) Two maize genes encoding omega-3 fatty acid desaturase and their differential expression to temperature.Plant Mol Biol 36: 297-306. https://doi.org/10.1023/a:1005993408270

5. BulgakovVP, AvramenkoTV (2020) Linking brassinosteroid and ABA signaling in the context of stress acclimation. Int J Mol Sci 21: 5108. https://doi.org/10.3390/ijms21145108

6. ChengCY, KrishnakumarV, ChanAP et al (2017) Araport11: a complete reannotation of the Arabidopsis thaliana reference genome. Plant J 89: 789-804. https://doi.org/10.1111/tpj.13415

7. Di C, Li M, Long F et al (2009) Molecular cloning, functional analysis and localization of a novel gene encoding polygalacturonase-inhibiting protein in Chorispora bungeana. Planta 231: 169-178. https://doi.org/10.1007/s00425-009-1039-7

8. EZ, Chen C, YangJ et al (2019) Genome-wide analysis of fatty acid desaturase genes in rice (Oryza sativa L.). Sci Rep 9:19445. https://doi.org/10.1038/s41598-019-55648-z

9. EreminaM, RozhonW, Poppenberger B (2016) Hormonal control of cold stress responses in plants. Cell Mol Life Sci 73:797-810. https://doi.org/10.1007/s00018-015-2089-6

10. Fu X, Chang J, An L et al. (2006) Association of the cold-hardiness of Chorispora bungeana with the distribution and accumulation of calcium in the cells and tissues. Environ Exp Bot 55:282-293. https://doi.org/10.1016/j.envexpbot.2004.11.009

11. Gibson S, Arondel V, Iba K et al (1994) Cloning of a temperature-regulated gene encoding a chloroplast omega-3 desaturase from Arabidopsis thaliana.Plant Physiol 106:1615-1621. https://doi.org/10.1104/pp.106.4.1615

12. Guan LL, WuW, HuB et al. (2014) Devolopmental and growth temperature regulation of omega-3 fatty acid desaturase genes in safflower (Carthamus tinctorius L.). Genet Mol Res 13:66236637. https://doi.org/10.4238/2014.August.28.7

13. Hu Y, JiangY, HanX, et al (2017) Jasmonate regulates leaf senescence and tolerance to cold stress: crosstalk with other phytohormones. J Exp Bot 68: 1361-1369. https://doi.org/10.1093/jxb/erx004

14. Iba K (2002) Acclimative response to temperature stress in higher plants: approaches of gene engineering for temperature tolerance. Annu Rev Plant Biol 53: 225245. https://doi.org/10.1146/annurev.arplant.53.100201.160729 
15. Iba K, Gibson S, Nishiuchi T et al. (1993) A gene encoding a chloroplast omega-3 fatty acid desaturase complements alterations in fatty acid desaturation and chloroplast copy number of the fad7 mutant of Arabidopsis thaliana. J Biol Chem 268: 24099-

24105. https://doi.org/10.1021/acs.jafc.0c05830

16. Iba K, Kodama H (1994) Omega-3 fatty acid desaturase genes and genetically engineered enhancement of cold tolerance in higher plants. Tanpakushitsu Kakusan Koso 39: 2803-2813.

17. JoH, KimM, ChoH et al. (2021) Identification of a potential gene for elevating $\omega-3$ concentration and its efficiency for improving the $\omega-6 / \omega-3$ ratio in soybean. J Agric Food Chem 69: 38363847. https://doi.org/10.1021/acs.jafc.0c05830

18. Karabudak T, Bor M, Özdemir F et al (2014) Glycine betaine protects tomato (Solanum lycopersicum) plants at low temperature by inducing fatty acid desaturase7 and lipoxygenase gene expression. Mol Biol Rep 41: 1401-1410. https://doi.org/10.1007/s11033-013-2984-6

19. KhanTA, FariduddinQ, YusufM (2017) Low-temperature stress: is phytohormones application a remedy? Environ Sci Pollut Res Int 24:21574-21590. https://doi.org/10.1007/s11356-017-9948-7

20. KimJA, Bhatnagar N, KwonSJ et al. (2018) Transcriptome analysis of ABA/JA-dual responsive genes in rice shoot and root. Curr Genomics 19: 4-

11. https://doi.org/ 10.2174/1389202918666170228134205.

21. KodamaH, Nishiuchi T, Seo $S$ et al (2000) Possible involvement of protein phosphorylation in the wound-responsive expression of Arabidopsis plastid omega-3 fatty acid desaturase gene. Plant Sci 155: 153-160. https://doi.org/10.1016/S0168-9452(00)00210-7

22. Ku YS, SintahaM, CheungMY et al (2018) Plant hormone signaling crosstalks between biotic and abiotic stress responses. Int J Mol Sci 19: 3206. https://doi.org/10.3390/ijms19103206

23. LiuW, LiW, HeQ et al (2015) Characterization of 19 genes encoding membrane-bound fatty acid desaturases and their expression profiles in Gossypium raimondii under low temperature. PLoS One 10: e0123281. https://doi.org/10.1371/journal.pone.0123281

24. Ma QP, You E, Wang J et al (2014) Isolation and expression of CsFAD7 and CsFAD8, two genes encoding $\omega-3$ fatty acid desaturase from Camellia sinensis. Acta Physiol Plant36: 23452352. $h$ ttps://doi.org/10.1007/s11738-014-1607-8

25. MatsudaO, Watanabe $\mathrm{C}$, Iba K (2001) Hormonal regulation of tissue-specific ectopic expression of an Arabidopsis endoplasmic reticulum-type omega-3 fatty acid desaturase (FAD3) gene. Planta 213:833-840. https://doi.org/10.1007/s004250100575

26. Martz F, Kiviniemi S, Palva TE et al (2006) Contribution of omega-3 fatty acid desaturase and 3ketoacyl-ACP synthase II (KASII) genes in the modulation of glycerolipid fatty acid composition during cold acclimation in birch leaves. J Exp Bot 57: 897-909. https://doi.org/10.1093/jxb/erj075

27. Matsuda O, Sakamoto H, Hashimoto T et al (2005) A temperature-sensitive mechanism that regulates post-translational stability of a plastidial omega-3 fatty acid desaturase (FAD8) in Arabidopsis leaf tissues.J Biol Chem 280: 3597-3604. https://doi.org/10.1074/jbc.M407226200 
28. McConnM, Hugly S, Browse J et al (1994) A mutation at the fad8 locus of Arabidopsis identifies a second chloroplast [omega]-3 desaturase. Plant Physiol 106: 1609-

1614. https://doi.org/10.1104/pp.106.4.1609

29. Mendes A, Kelly AA, Erp H et al. (2013) bZIP67 regulates the omega-3 fatty acid content of Arabidopsis seed oil by activating fatty acid desaturase3. Plant Cell 25: $3104-$ 3116. https://doi.org/10.1105/tpc. 113.116343

30. Mikami K, Murata N (2003) Membrane fluidity and the perception of environmental signals in cyanobacteria and plants. Prog Lipid Res 42: 527-543. https://doi.org/10.1016/S01637827(03)00036-5

31. NishiuchiT, Kodama H, Yanagisawa S et al (1999) Wound-induced expression of the FAD7 gene is mediated by different regulatory domains of its promoter in leaves/stems and roots. Plant Physiol 121: 1239-1246. https://doi.org/10.1104/pp.121.4.1239

32. NorlinaR, Norashikin MN, LohSH, et al (2020) Exogenous abscisic acid supplementation at early stationary growth phase triggers changes in the regulation of fatty acid biosynthesis in Chlorella vulgaris UMT-M1. Appl Biochem Biotechnol191: 1653-1669. https://doi.org/10.1007/s12010-02003312-y

33. Penfield S (2008) Temperature perception and signal transduction in plants. New Phytol 179: 615628. $h$ ttps://doi.org/10.1111/j.1469-8137.2008.02478.x

34. RamirezVE, Poppenberger B (2020) Modes of brassinosteroid activity in cold stress tolerance. Front Plant Sci 11: 583666. https://doi.org/10.3389/fp/s.2020.583666

35. Román Á, Andreu V, Hernández ML et al. (2012) Contribution of the different omega-3 fatty acid desaturase genes to the cold response in soybean. J Exp Bot 63: 49734982. https://doi.org/10.1093/jxb/ers174

36. Román Á, Hernández ML, Soria-García Á et al. (2015) Non-redundant contribution of the plastidial FAD8 $\omega$-3 desaturase to glycerolipid unsaturation at different temperatures in Arabidopsis. Mol Plant 8: 15599-15611. https://doi.org/10.1016/j.molp.2015.06.004

37. Routaboul JM, Fischer SF, Browse J (2000) Trienoic fatty acids are required to maintain chloroplast function at low temperatures. Plant Physiol 124: 1697-1705. https://doi.org/10.1104/pp.124.4.1697

38. Shi Y, An L, Zhang M et al (2008) Regulation of the plasma membrane during exposure to low temperatures in suspension-cultured cells from a cryophyte (Chorispora bungeana). Protoplasma 232: 173-181. https://doi.org/10.1007/s00709-008-0291-1

39. Shi Y, An L, Li X et al (2011) The octadecanoid signaling pathway participates in the chilling-induced transcription of $\omega-3$ fatty acid desaturases in Arabidopsis. Plant Physiol Biochem 49: 208215. https://doi.org/10.1016/j.plaphy.2010.11.013

40. Shi Y, DingY, YangS (2015) Cold signal transduction and its interplay with phytohormones during cold acclimation. Plant Cell Physiol 56: 7-15. https://doi.org/10.1093/pcp/pcu115

41. ShiY, YueX, $A n L$ (2018) Integrated regulation triggered by a cryophyte $\omega-3$ desaturase gene confers multiple-stress tolerance in tobacco. J Exp Bot 69: 2131-2148. https://doi.org/10.1093/jxb/ery050 
42. Song C, Yang Y, Yang T et al. (2019) MaMYB4 recruits histone deacetylase MaHDA2 and modulates the expression of $\omega-3$ fatty acid desaturase genes during cold stress response in banana fruit. Plant Cell Physiol 60: 2410-2422. https://doi.org/10.1093/pcp/pcz142

43. Soria-García A, Rubio MC, Lagunas B et al. (2019) Tissue distribution and specific contribution of Arabidopsis FAD7 and FAD8 plastid desaturases to the JA- and ABA-Mediated cold stress or defense responses. Plant Cell Physiol 60: 1025-1040. https://doi.org/10.1093/pcp/pcz017

44. Tang S, Guan R, Zhang H et al(2007)Cloning and expression analysis of three cDNAs encoding omega-3 fatty acid desaturases from Descurainiasophia.Biotechnol Lett 29: 14171424. $h$ ttps://doi.org/10.1007/s10529-007-9391-9

45. Teixeira MC, Carvalho IS, Brodelius M (2010) Omega-3 fatty acid desaturase genes isolated from purslane (Portulaca oleracea L.): expression in different tissues and response to cold and wound stress. J Agric Food Chem 58: 1870-1877. https://doi.org/10.1021/jf902684v

46. TovuuA, ZulfugarovIS, WuG et al. (2016) Rice mutants deficient in $\omega-3$ fatty acid desaturase (FAD8) fail to acclimate to cold temperatures. Plant Physiol Biochem 109: 525535. https://doi.org/10.1016/j.plaphy.2016.11.001

47. Upchurch RG (2008) Fatty acid unsaturation, mobilization, and regulation in the response of plants to stress.Biotechnol Lett 30: 967-977. https://doi.org/10.1007/s10529-008-9639-z

48. Verma V, Ravindran P, Kumar PP (2016) Plant hormone-mediated regulation of stress responses. BMC Plant Biol 16: 86. https://doi.org/10.1186/s12870-016-0771-y

49. Vijayan P, Browse J (2002) Photoinhibition in mutants of Arabidopsis deficient in thylakoid unsaturation. Plant Physiol 129: 876-885. https://doi.org/10.1104/pp.004341

50. Wada H, Murata N (2007) The essential role of phosphatidylglycerol in photosynthesis. Photosynth Res 92: 205-215. https://doi.org/10.1007/s11120-007-9203-z

51. Wang J, Ming F, Pittman Jet al. Characterization of a rice (Oryza sativa L.) gene encoding a temperature-dependent chloroplast omega-3 fatty acid desaturase. Biochem Biophys Res Commun340: 1209-1216. https://doi.org/10.1016/j.bbrc.2005.12.126

52. Wu J, Qu T, Chen S et al (2009) Molecular cloning and characterization of a gammaglutamylcysteine synthetase gene from Chorispora bungeana. Protoplasma 235: 2736. $h$ ttps://doi.org/10.1007/s00709-008-0026-3

53. Yang J, Duan G, Li C et al. (2019) The crosstalks between jasmonic acid and other plant hormone signaling highlight the involvement of jasmonic acid as a core component in plant response to biotic and abiotic stresses. Front Plant Sci 10: 1349. https://doi.org/10.3389/fp/s.2019.01349

54. Yurchenko OP, Park S, llut DC et al. (2014) Genome-wide analysis of the omega-3 fatty acid desaturase gene family in Gossypium. BMC Plant Biol 14: 312. https://doi.org/10.1186/s12870-0140312-5

55. WangJ, SongL, Gong $X$ et al (2020) Functions of jasmonic acid in plant regulation and response to abiotic stress. Int J Mol Sci21: 1446. https://doi.org/10.3390/ijms21041446 
56. YueX, ZhangG, ZhaoZ et al. (2019) A cryophyte transcription factor, CbABF1, confers freezing, and drought tolerance in tobacco. Front Plant Sci10: 699. https://doi.org/10.3389/fp/s.2019.00699

57. ZhangZ, WeiX, LiuW et al. (2018) Genome-wide identification and expression analysis of the fatty acid desaturase genes in Medicago truncatula. Biochem. Biophys Res Commun 499: 361367. https://doi.org/10.1016/j.bbrc.2018.03.165

58. Zhang L, Si J, Zeng F et al (2009) Molecular cloning and characterization of a ferritin gene upregulated by cold stress in Chorispora bungeana. Biol Trace Elem Res 128: 269283. https://doi.org/10.1007/s12011-008-8275-8

59. Zhang M, Barg R, Yin M et al. (2005) Modulated fatty acid desaturation via overexpression of two distinct omega-3 desaturases differentially alters tolerance to various abiotic stresses in transgenic tobacco cells and plants. Plant J 44: 361-371. https://doi.org/10.1111/j.1365-313X.2005.02536.X

60. Zhang T, Liu Y, Xue L et al. (2006) Molecular cloning and characterization of a novel MAP kinase gene in Chorispora bungeana. Plant Physiol Biochem 44: 78-

84. https://doi.org/10.1016/j.plaphy.2006.01.001

61. Zhang YM, Wang CC, Hu HH et al (2011) Cloning and expression of three fatty acid desaturase genes from cold-sensitive lima bean (Phaseolus lunatus L.). Biotechnol Lett 33: 395-

401. https://doi.org/10.1007/s10529-010-0432-4

62. Zhao Z, Tan L, Dang C et al (2012) Deep-sequencing transcriptome analysis of chilling tolerance mechanisms of a subnival alpine plant, Chorispora bungeana. BMC Plant Biol12:

222.https://doi.org/10.1186/1471-2229-12-222

\section{Tables}

Table 1. Pearson correlation coefficients (two-tailed) between the cold-responsive and the hormoneregulated expressions of $\omega-3$ CbFAD genes in $C$. bungeanacells.

\begin{tabular}{|c|c|c|c|c|c|c|}
\hline Gene name & Temperature $\left({ }^{\circ} \mathrm{C}\right)$ & SA & BRs & $J A$ & ABA & GA3 \\
\hline \multirow[t]{3}{*}{ CbFAD3 } & 4 & -0.26 & $0.99 * \star \star$ & 0.07 & 0.04 & -0.55 \\
\hline & 0 & -0.35 & $0.97 * \star \star$ & -0.07 & -0.16 & -0.67 \\
\hline & -4 & -0.94 & 0.35 & $0.51 *$ & -0.09 & 0.10 \\
\hline \multirow[t]{3}{*}{ CbFAD7 } & 4 & -0.02 & 0.78 ** & $0.94 * \star$ & 0.22 & -0.65 \\
\hline & 0 & 0.23 & $0.91^{\star \star \star}$ & $0.72^{\star *}$ & -0.14 & -0.64 \\
\hline & -4 & -0.61 & 0.48 & $0.82^{\star \star \star}$ & -0.23 & -0.78 \\
\hline \multirow[t]{3}{*}{ CbFAD8 } & 4 & -0.73 & $0.72^{* *}$ & 0.71 ** & -0.75 & -0.34 \\
\hline & 0 & -0.16 & $0.99 * \star \star$ & $0.97^{* \star \star}$ & -0.26 & -0.60 \\
\hline & -4 & 0.02 & $0.91 * \star \star$ & $0.87 * \star \star$ & 0.00 & -0.83 \\
\hline
\end{tabular}


Three replications for each sample, $n=5 \times 3$. Significant positive correlation $(R>0.50, P<0.05)$ is indicated in bold. * $P<0.05, * \star P<0.01, * \star \star P<0.001$.

Table 2. Pearson correlation coefficients (two-tailed) between the cold-responsive and the hormoneregulated expressions of $\omega-3$ CbFAD genes in $C$. bungeana leaves.

\begin{tabular}{|c|c|c|c|c|c|}
\hline Gene name & Temperature $\left({ }^{\circ} \mathrm{C}\right)$ & BRs & JA & ABA & GA3 \\
\hline \multirow[t]{3}{*}{ CbFAD3 } & 4 & $0.85^{\star \star \star}$ & $0.60 *$ & $0.90 * \star \star$ & 0.78 ** \\
\hline & 0 & 0.23 & $0.99 * \star \star$ & $0.86 * \star \star$ & $0.71 * \star$ \\
\hline & -4 & 0.22 & $0.95^{\star \star *}$ & 0.91 ** & $0.82^{* \star \star}$ \\
\hline \multirow[t]{3}{*}{ CbFAD7 } & 4 & $0.94 * * *$ & -0.24 & 0.47 & $0.86 * * \star$ \\
\hline & 0 & $0.93^{* \star *}$ & -0.29 & 0.49 & $0.95^{\star \star \star}$ \\
\hline & -4 & $0.90 * \star *$ & -0.77 & 0.46 & $0.74 * \star$ \\
\hline \multirow[t]{3}{*}{ CbFAD8 } & 4 & -0.76 & 0.36 & 0.39 & $0.69 *$ \\
\hline & 0 & -0.21 & $0.97 * \star \star$ & $0.97^{\star \star \star}$ & -0.11 \\
\hline & -4 & -0.08 & $0.98 * \star *$ & $0.98 * \star *$ & -0.29 \\
\hline
\end{tabular}

Three replications for each sample, $n=5 \times 3$. Significant positive correlation $(R>0.50, P<0.05)$ is indicated in bold. * $P<0.05, * \star P<0.01$, *** $P<0.001$.

\section{Supplementary Figures And Tables}

Supplementary Figures and Tables are not available with this version.

\section{Figures}


b

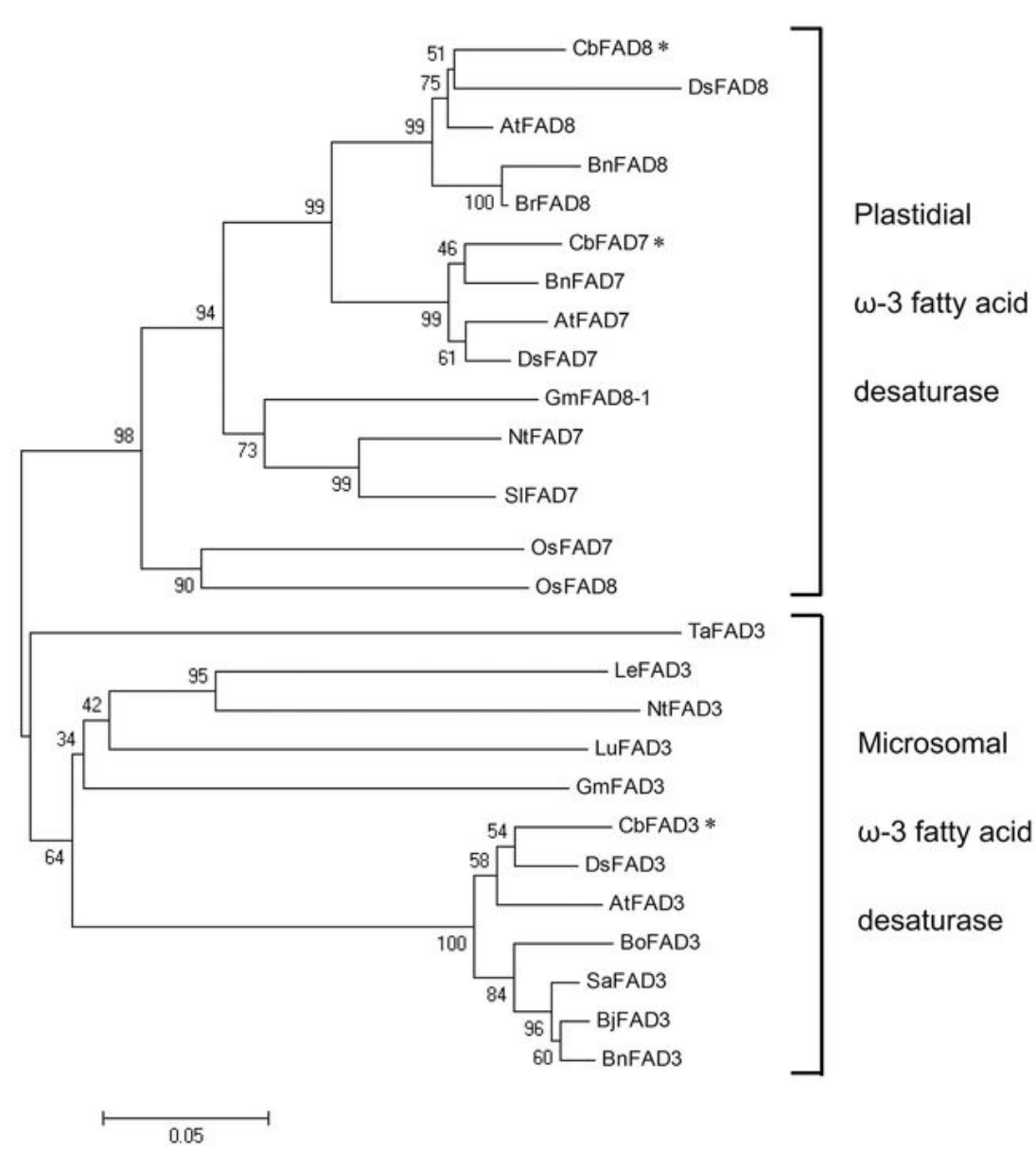

\section{Figure 1}

Analysis of the deduced amino acid sequences of C. bungeana $\omega-3$ FADs. (a) Sequence comparison of CbFAD3, CbFAD7 and CbFAD8. Identical and similar residues are shown on a background of black and gray, respectively. The sequences of the putative chloroplast transit peptides of CbFAD7 and CbFAD8 are arrowed. The three conserved histidine clusters are indicated by asterisks, and the four transmembrane domains (TMD) are underlined. (b) Phylogenetic tree analysis of CbFAD3, CbFAD7 and CbFAD8. The 
positions of $\omega-3$ CbFADs are asterisked. The accession number of different $\omega-3$ FADs included in this analysis: Arabidopsis thaliana AtFAD3 (NP180559), Brassica juncea BjFAD3 (ADJ58019), Brassica napus BnFAD3 (NP001302640), Brassica oleracea BoFAD3 (AGH20189), Chorispora bungeana CbFAD3 (KM591203), Chorispora bungeana CbFAD7 (KY069282), Chorispora bungeana CbFAD8 (KY069283), Descurainia sophia DsFAD3 (ABK91879), Glycine max GmFAD3 (NP001237507), Lycopersicon esculentum LeFAD3 (ABX24525), Linum usitatissimum LuFAD3 (AFJ53089), Nicotiana tabacum NtFAD3 (P48626), Sinapis alba SaFAD3 (AHA05997), Triticum aestivum TaFAD3 (BAA28358), Arabidopsis thaliana AtFAD7 (P46310), Brassica napus BnFAD7 (ACS26170), Descurainia sophia DsFAD7 (ABS86961), Nicotiana tabacum NtFAD7 (D79979), Solanum lycopersicum SIFAD7 (NP001234592), Oryza sativa OsFAD7 (BAE79783), Arabidopsis thaliana AtFAD8 (P48622), Brassica napus BnFAD8 (NP001302644), Brassica rapa BrFAD8 (AAW78909), Descurainia sophia DsFAD8 (ABK91881), Glycine max GmFAD8-1 (NP001238609), Oryza sativa OsFAD8 (BAE79784).
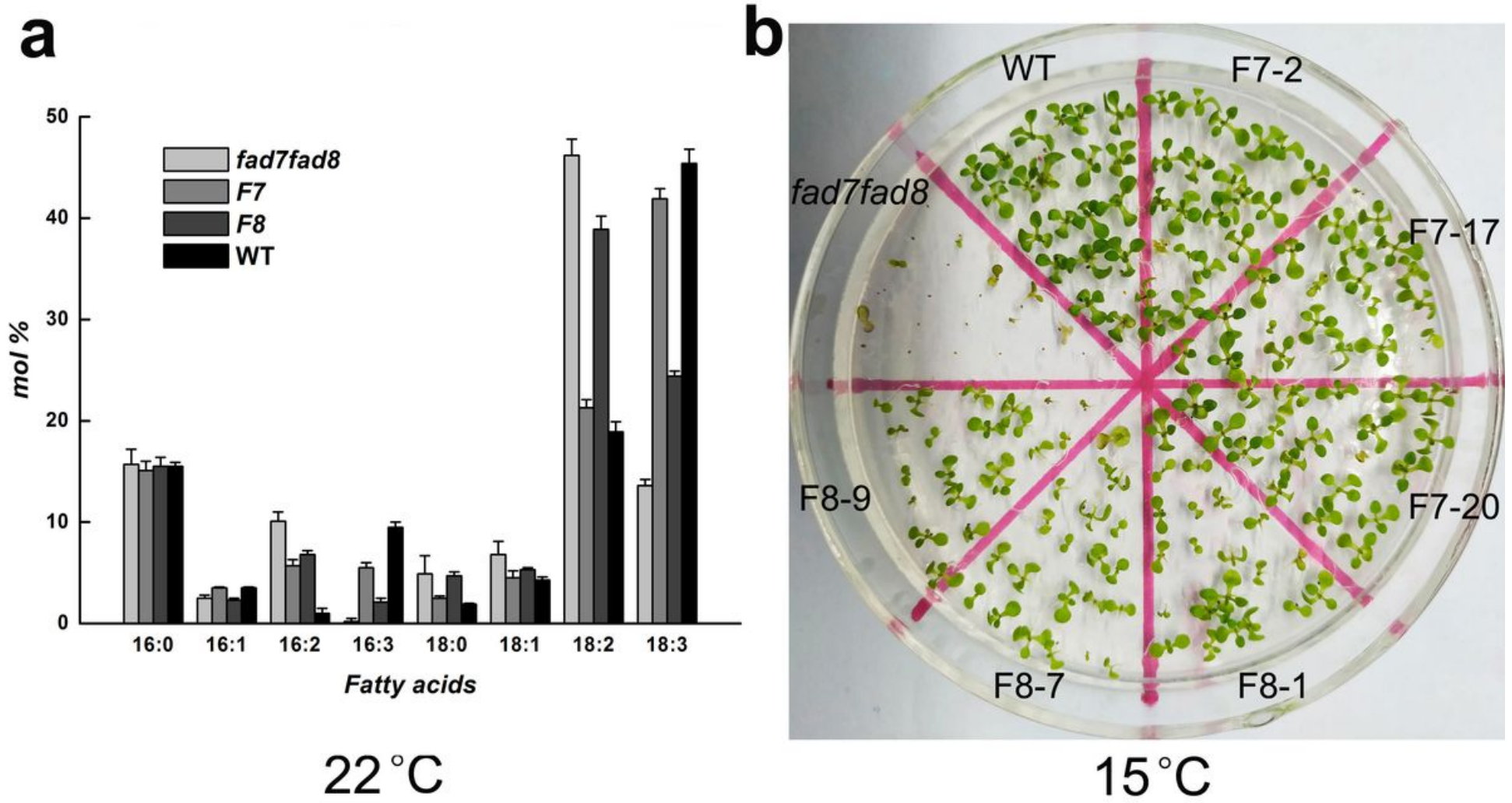

Figure 2

Functionality of CbFAD7 and CbFAD8 were verified in Arabidopsis mutant. (a) Comparison of total leaf fatty acids between Arabidopsis lines under normal conditions. (b) Comparison of low-temperature germination between Arabidopsis lines. WT means wild type (Col-0); fad7fad8 means double fad7fad8 mutant; F7 means CbFAD7-complemented mutant; F8 means CbFAD8-complemented mutant. Each value represents the mean \pm SE of five replicates. 

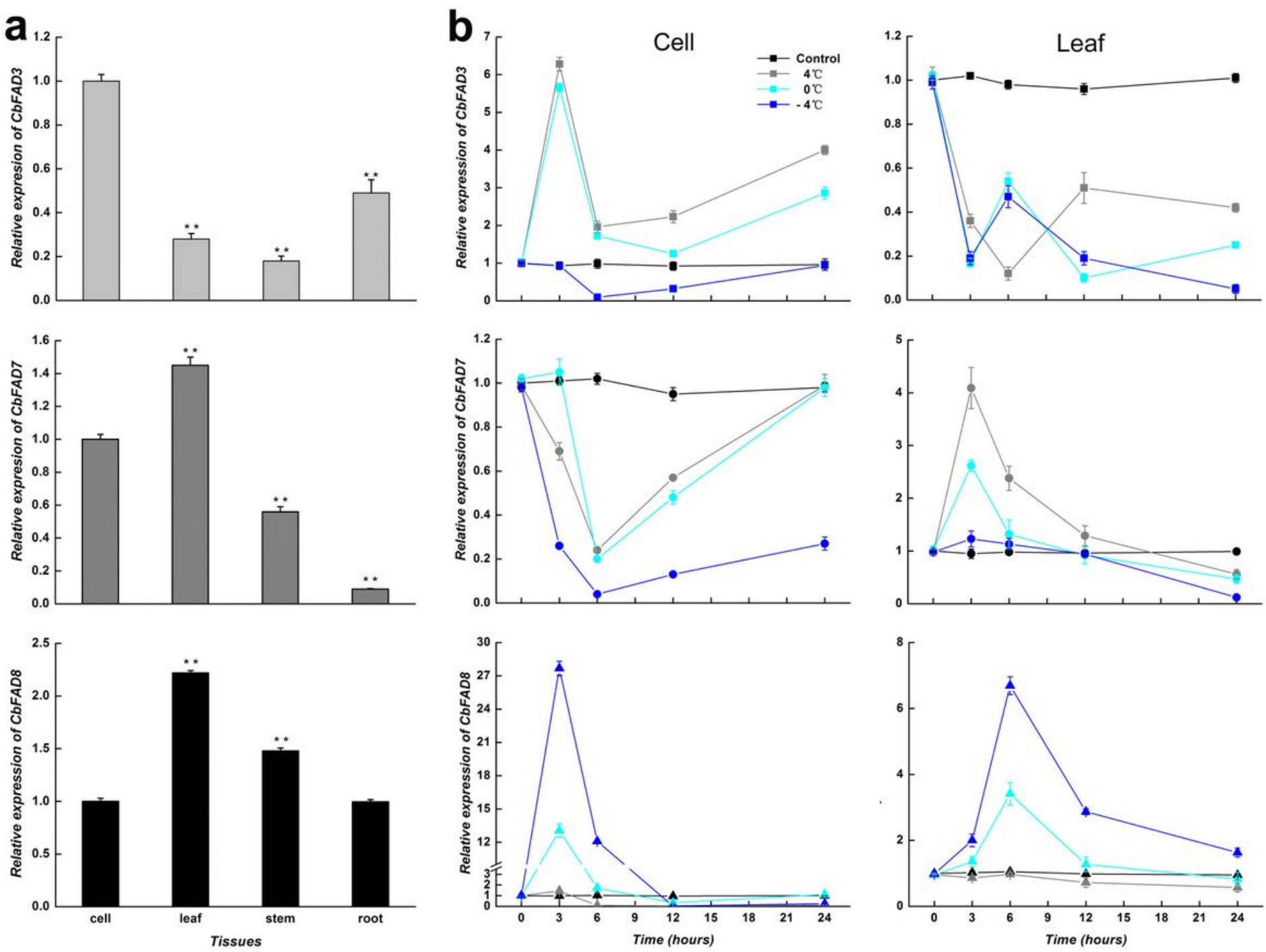

Figure 3

Expression patterns of $\omega-3$ CbFAD genes in C. bungeana. (a) Tissue-specific expressions of CbFAD3, CbFAD7 and CbFAD8. Data were presented as relative expression ratios being compared with the expression levels of corresponding genes in suspension-cultured cells, which were set at a value of 1 . (b) Cold-responsive expressions of CbFAD3, CbFAD7 and CbFAD8 in cell suspensions and plant leaves. Data were presented as relative expression ratios being compared with the expression levels of corresponding genes before treatment $(0 \mathrm{~h})$, which were set at a value of 1 . Each value represents the mean $\pm \mathrm{SE}$ of three replicates. 

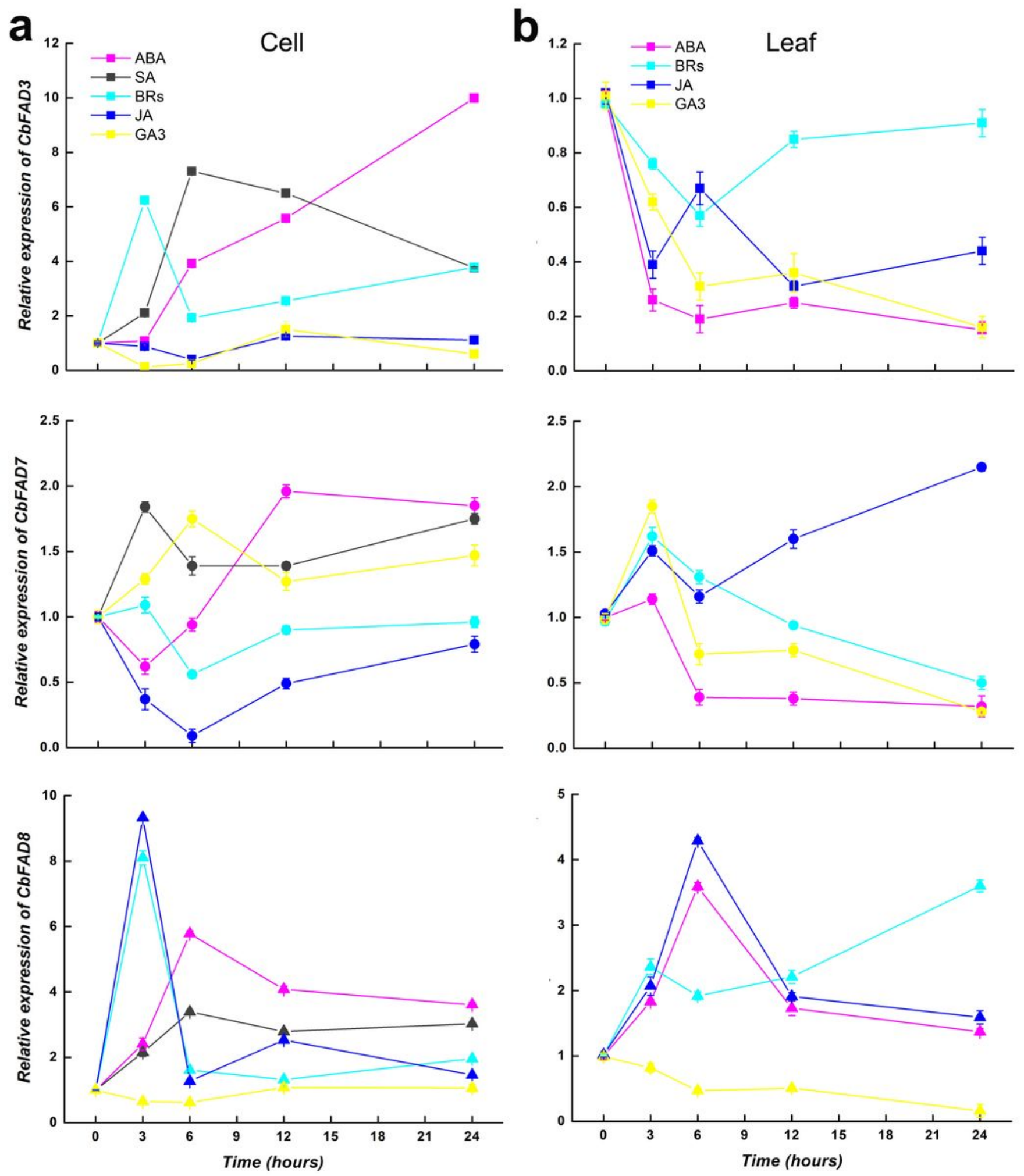

Figure 4

Hormone-responsive expressions of $\omega-3$ CbFAD genes in C. bungeana. (a) Expression patterns of CbFAD3, CbFAD7 and CbFAD8 in suspension-cultured cells. (b) Expression patterns of CbFAD3, CbFAD7 and CbFAD8 in plant leaves. Data were presented as relative expression ratios being compared with the expression levels of corresponding genes before treatment $(0 \mathrm{~h})$, which were set at a value of 1 . Each value represents the mean \pm SE of three replicates. 

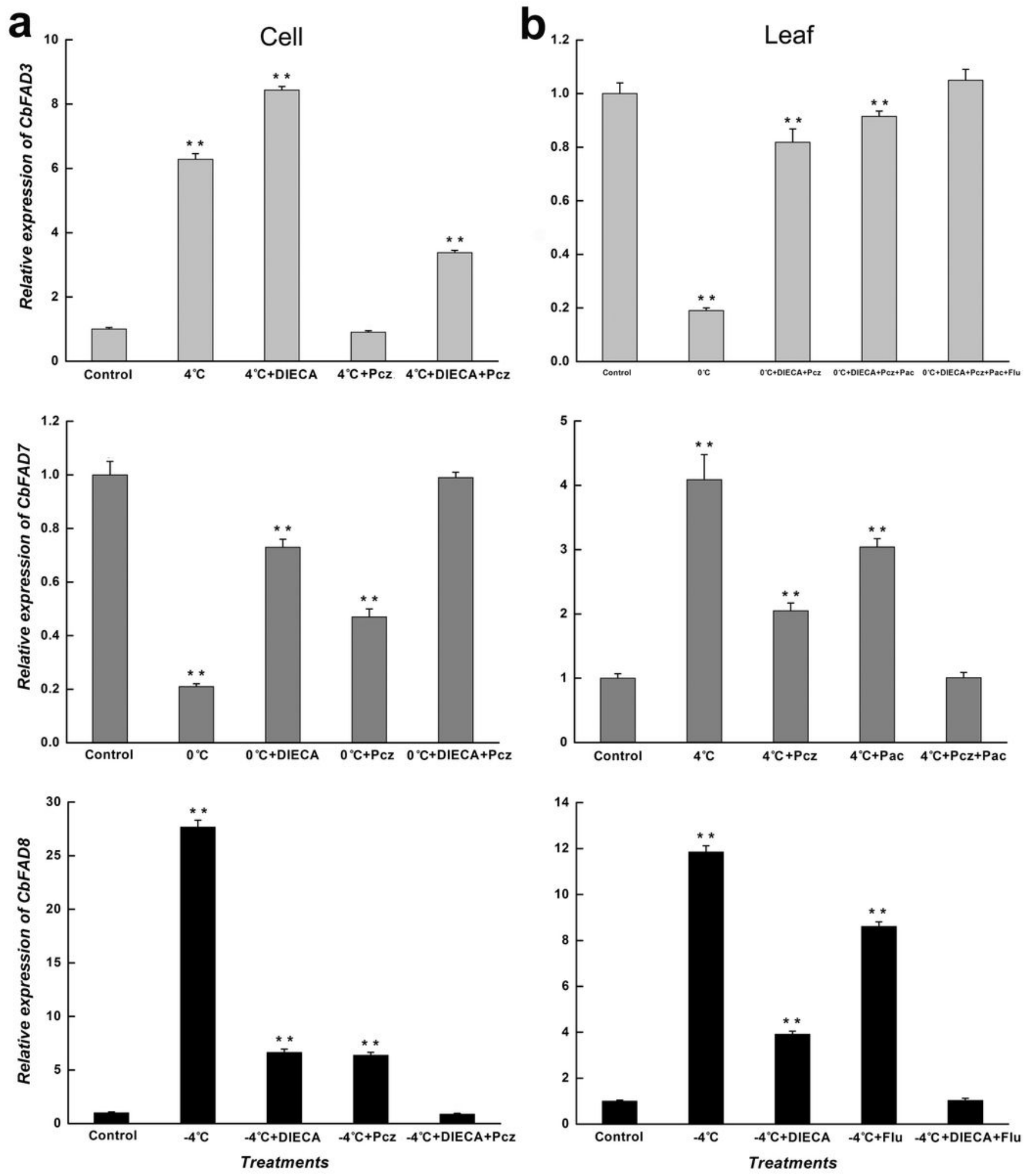

Figure 5

Effects of hormone inhibitors on the cold-responsive expressions of $\omega-3$ CbFAD genes in $C$. bungeana. (a) Expression levels of CbFAD3, CbFAD7 and CbFAD8 in suspension-cultured cells. The expressions of CbFAD3 and CbFAD8 were detected at being treated for $3 \mathrm{~h}$, while that of CbFAD7 was detected at being treated for $6 \mathrm{~h}$. (b) Expression levels of CbFAD3, CbFAD7 and CbFAD8 in plant leaves. The expressions of CbFAD3 and CbFAD7 were detected at being treated for $3 \mathrm{~h}$, while that of CbFAD 8 was detected at being 
treated for $6 \mathrm{~h}$. Data were presented as relative expression ratios being compared with the expression levels of corresponding genes under normal conditions (Control), which were set at a value of 1 . Each value represents the mean \pm SE of three replicates.
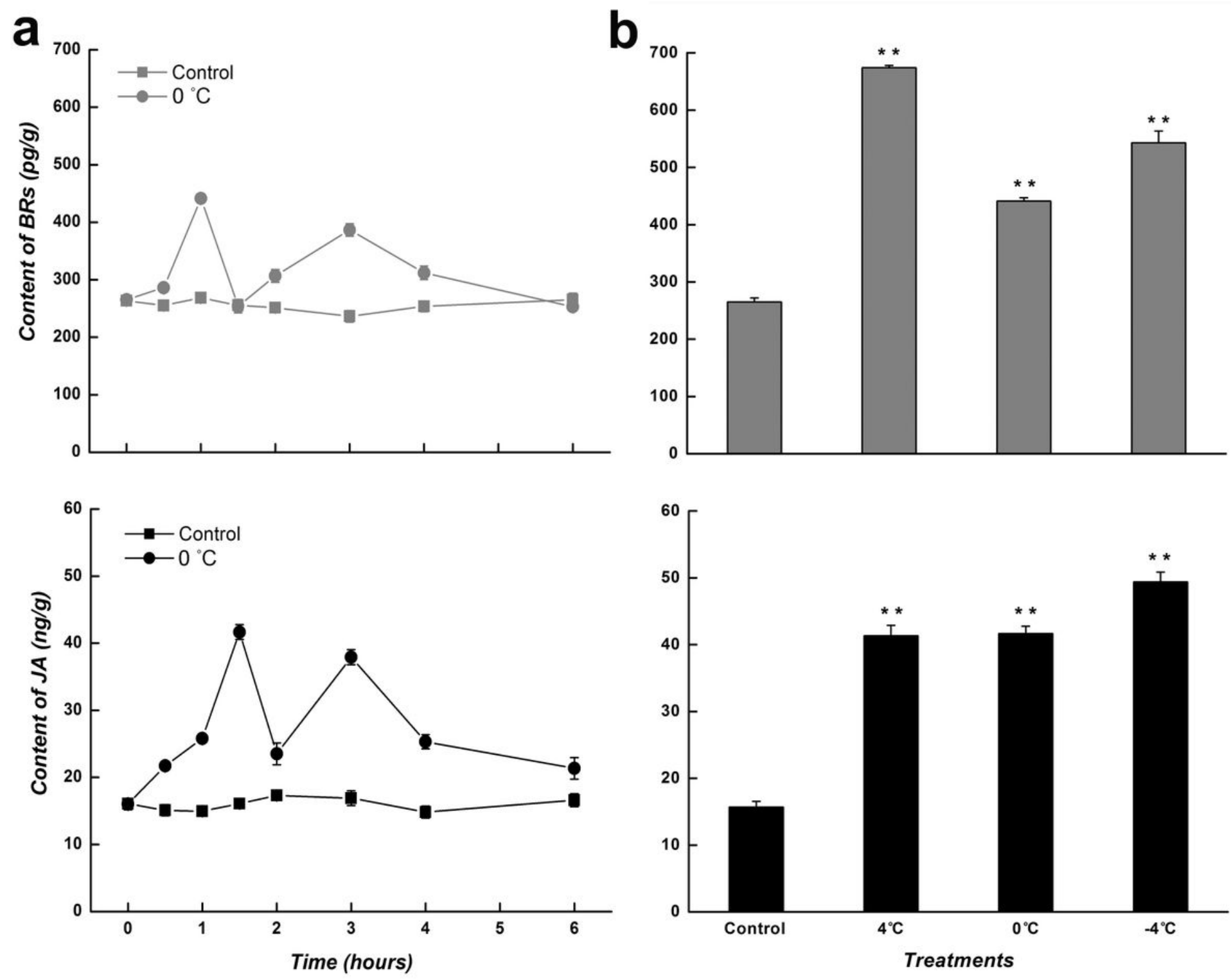

Figure 6

Pytohormone analysis in the suspension-cultured cells of $\mathrm{C}$. bungeana at low temperatures. (a) Changes in the level of BRs and JA at $0{ }^{\circ} \mathrm{C}$ (b) Levels of BRs and JA at different low temperatures $\left(4,0\right.$ and $\left.-4{ }^{\circ} \mathrm{C}\right)$. The data of BRs was detected at being treated for $1 \mathrm{~h}$, and that of JA was detected at being treated for $1.5 \mathrm{~h}$. The corresponding data at the same time point under normal conditions was taken as the control. Each value represents the mean \pm SE of five replicates. 

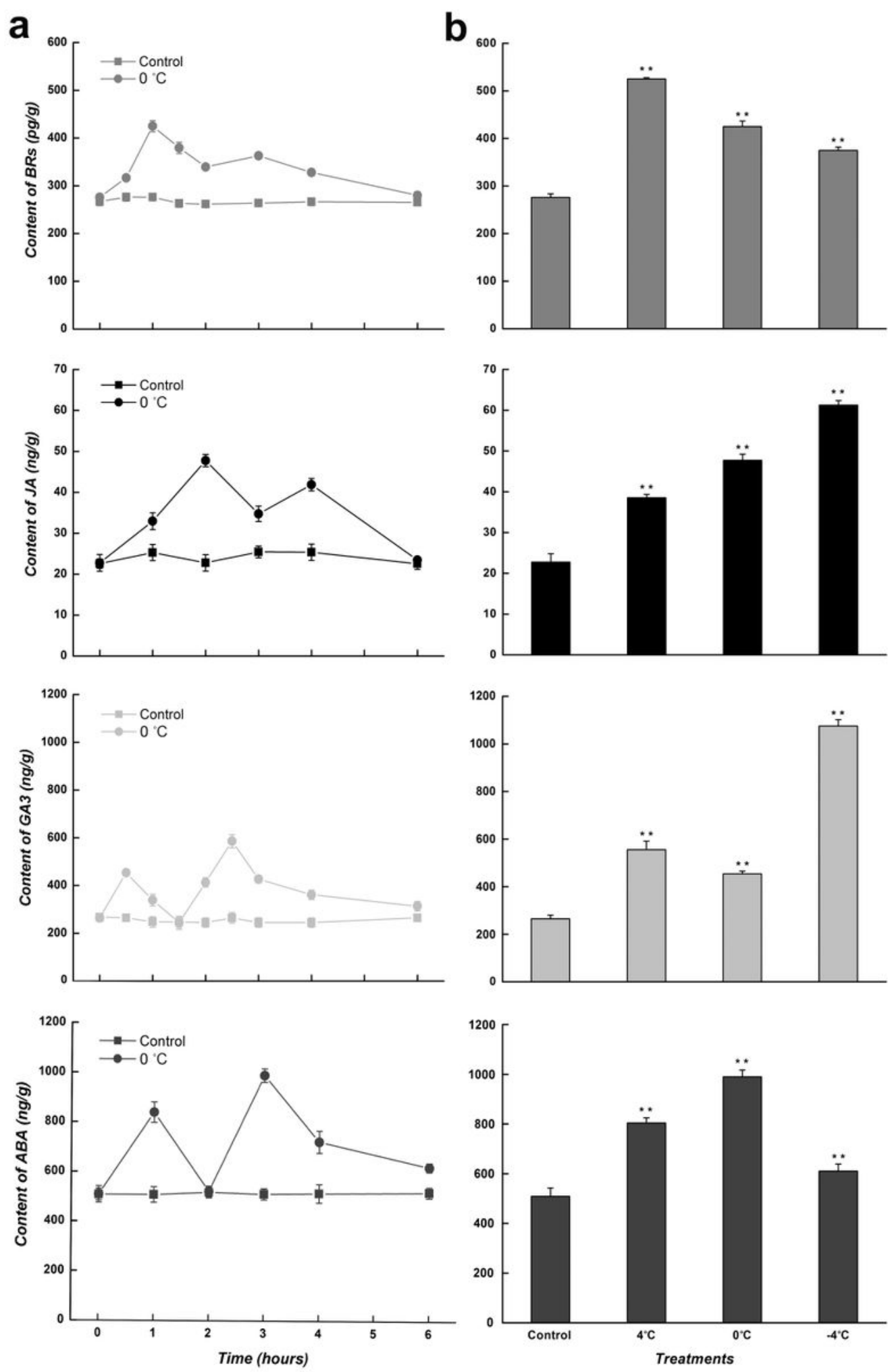

Figure 7

Pytohormone analysis in the plant leaves of $\mathrm{C}$. bungeana at low temperatures. (a) Changes in the level of BRs, JA, GA3 and ABA at $0{ }^{\circ} \mathrm{C}$. (b) Levels of BRs, JA, GA3 and ABA at different low temperatures $(4,0$ and $-4{ }^{\circ} \mathrm{C}$ ). The data of BRs and ABA were detected at being treated for $1 \mathrm{~h}$, and those of JA and GA3 were detected at being treated for 2 and $0.5 \mathrm{~h}$, respectively. The corresponding data at the same time point under normal conditions was taken as the control. Each value represents the mean \pm SE of five replicates. 


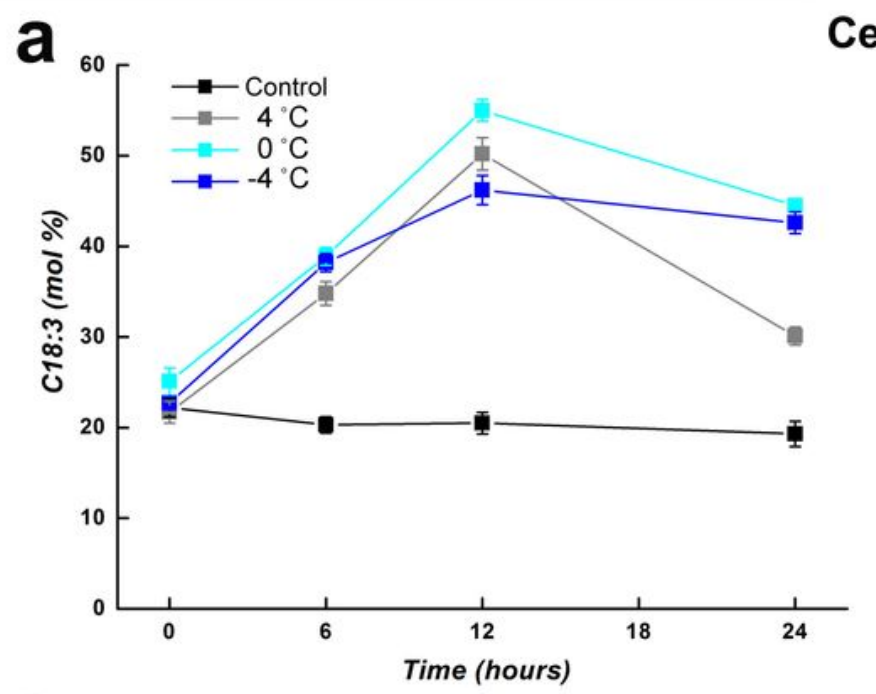

\section{Cell}
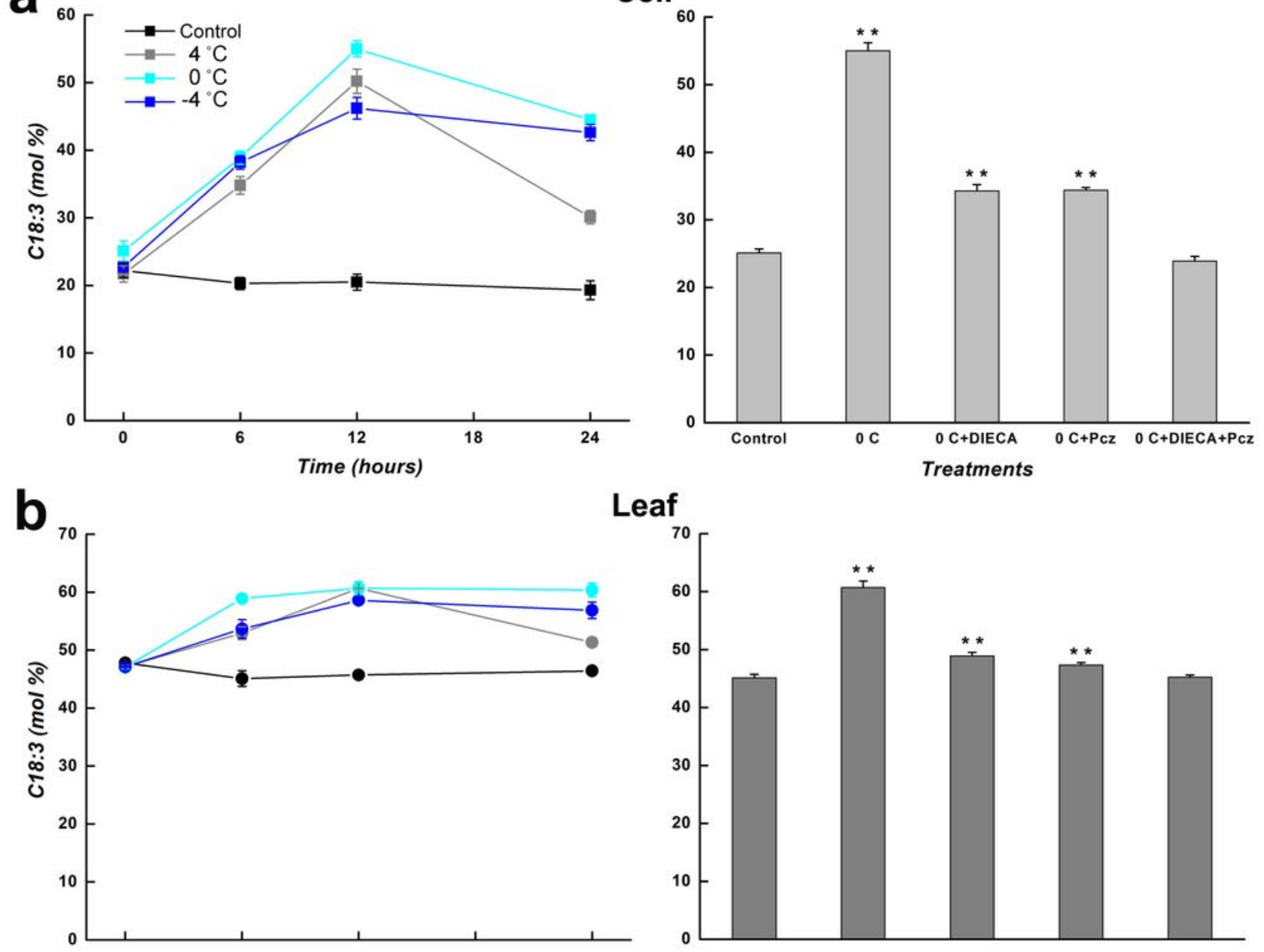

Leaf
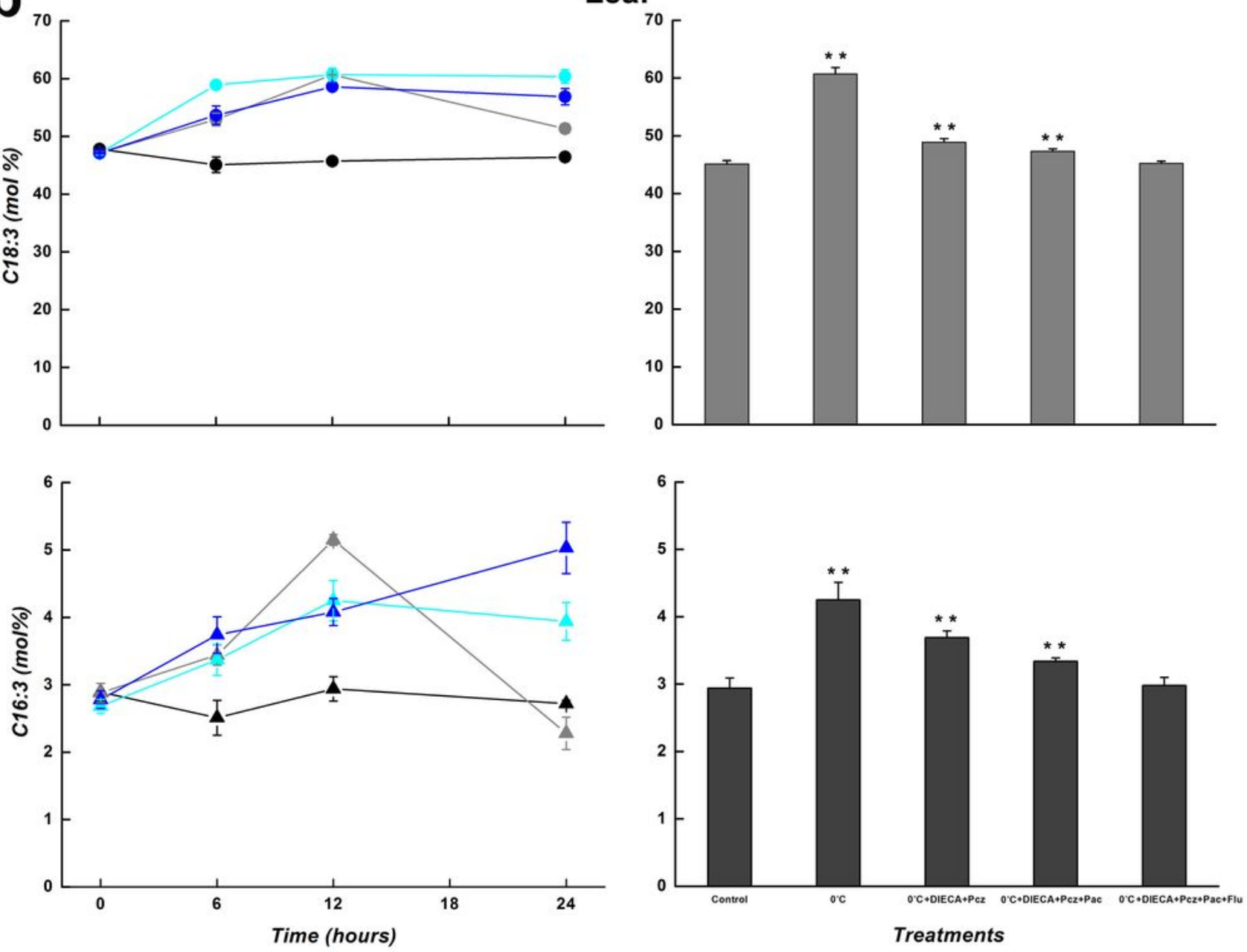

Figure 8

Levels of TAs in C. bungeana under low-temperature and hormone-inhibition treatments. (a) Levels of C18:3 in the total lipids of suspension-cultured cells. (b) Levels of C18:3 and C16:3 in the total lipids of plant leaves. The data of inhibition treatments were detected at being treated for $12 \mathrm{~h}$. The corresponding data at the same time point under normal conditions was taken as the control. Each value represents the mean \pm SE of five replicates. 